\title{
Photoacoustic elastography imaging: a review
}

Mayanglambam Suheshkumar Singh Anjali Thomas 


\title{
Photoacoustic elastography imaging: a review
}

\author{
Mayanglambam Suheshkumar Singh* and Anjali Thomas \\ Indian Institute of Science Education and Research Thiruvananthapuram (IISER-TVM), School of Physics (SoP), Biomedical Instrumentation and \\ Imaging Laboratory (BIIL), Thiruvananthapuram, Kerala, India
}

\begin{abstract}
Elastography imaging is a promising tool-in both research and clinical settings-for diagnosis, staging, and therapeutic treatments of various life-threatening diseases (including brain tumors, breast cancers, prostate cancers, and Alzheimer's disease). Large variation in the physical (elastic) properties of tissue, from normal to diseased stages, enables highly sensitive characterization of pathophysiological states of the diseases. On the other hand, over the last decade or so, photoacoustic (PA) imaging —an imaging modality that combines the advantageous features of two separate imaging modalities, i.e., high spatial resolution and high contrast obtainable, respectively, from ultrasound- and optical-based modalities-has been emerging and widely studied. Recently, recovery of elastic properties of soft biological tissues-in addition to prior reported recovery of vital tissue physiological information $\left(\mathrm{Hb}, \mathrm{HbO}_{2}, \mathrm{SO}\right.$, and total $\left.\mathrm{Hb}\right)$, noninvasively and nondestructively, with unprecedented spatial resolution $(\mu \mathrm{m})$ at penetration depth $(\mathrm{cm})$ - has been reported. Studies demonstrating that combined recovery of mechanical tissue properties and physiological information-by a single (PA) imaging unit-pave a promising platform in clinical diagnosis and therapeutic treatments. We offer a comprehensive review of PA imaging technology, focusing on recent advances in relation to elastography. Our review draws out technological challenges pertaining to PA elastography (PAE) imaging, and viable approaches. Currently, PAE imaging is in the nurture stage of its development, where the technology is limited to qualitative study. The prevailing challenges (specifically, quantitative measurements) may be addressed in a similar way by which ultrasound elastography and optical coherence elastography were accredited for quantitative measurements. (C) The Authors. Published by SPIE under a Creative Commons Attribution 4.0 Unported License. Distribution or reproduction of this work in whole or in part requires full attribution of the original publication, including its DOI. [DOI: 10.1117/1.JBO.24.4.040902]
\end{abstract}

Keywords: elastography; photoacoustic imaging; soft tissues; physiological activities; diagnosis; therapeutic treatments.

Paper 180652VRR received Dec. 8, 2018; accepted for publication Apr. 5, 2019; published online Apr. $30,2019$.

\section{Introduction}

Evaluation of the elastic properties of tissues offers a promising tool for diagnosis, staging, and therapeutic treatments (coupled with continuous monitoring) of several life-threatening diseases, including cancers. ${ }^{1,2}$ Mechanical elasticity or stiffness of soft biological tissues is dependent on the microscopic and/or macroscopic structural organization of their constituent molecules, ${ }^{2-5}$ whereas changes in the stromal density and molecular texture of biological tissues - which are closely associated with changes in physiological activities resulting from development and progression of the diseases-are correlated with pathophysiological states of tissues. ${ }^{6-9}$ In other words, change in mechanical properties (i.e., hardness) of diseased tissues relative to normal background tissue accompanies the development of various lumps and cancerous lesions (many folds ${ }^{4} \sim 50$ ). Thus, characterization and/or imaging of tissue hardness facilitates a sensitive way (with good contrast) to detect structural changes abnormally deep inside tissue; thus, these changes can be quantified in terms of characteristic (tissue) elastic coefficients, i.e., shear or Young's modulus, whereas changes in bulk modulus remain insignificant for soft tissues. ${ }^{9}$

Manual palpation is an ancient technique ${ }^{2}$ that was devised to detect a hard lump (superficial or deep-seated) in a body. Technically, in this manual technique, external mechanical pressure is applied (remotely) deep inside the soft tissue of interest

*Address all correspondence to Mayanglambam Suheshkumar Singh, E-mail: suhesh.kumar@iisertvm.ac.in by gently pressing over the skin of a patient; in turn, the response of the tissue subjected to the mechanical strain exerted by the physician's hand is examined upon detection. Briefly, manual palpation is a qualitative study of textural difference of lesion relative to background tissue. ${ }^{9}$ This manual technique suffers from serious drawbacks: ${ }^{4,9}(1)$ it is subjective, i.e., diagnosis of diseases is entirely dependent on experience of the examining medical practitioner, (2) it fails to detect and, hence, fails to investigate smaller and/or deeper tumors that are key factors for early diagnosis and successful therapeutic treatments, and (3) it provides only qualitative (not quantitative) diagnostic information. In view of addressing the issues pertaining to manual palpation, elastography imaging techniques have been improvised over the last three decades or so ${ }^{6,9,10}$ for recovery and imaging — both qualitatively and quantitatively ${ }^{10-61}$ - of the elastic modulus of soft biological tissues (for various medical applications). As mentioned in Ref. 62, elastography imaging was first studied (in 1991) by Ophir et al. ${ }^{6}$ employing ultrasound imaging technique. In the reported study, 2-D images representative of elastic modulus distribution of tissues were presented. Since then, several nondestructive imaging modalities [including ultrasound, ${ }^{11-13}$ magnetic resonance imaging (MRl), ${ }^{10,14-21}$ optical coherence tomography (OCT), ${ }^{22-46}$ computed tomography (CT) scans, ${ }^{47-50}$ speckle contrast,,${ }^{51-58}$ and ultrasound modulated optical tomography (UMOT) ${ }^{59-61}$ ] and several techniques (static and dynamic) have been reported in the literature. In Ref. 63, a historical roadmap perspective of the 20-year journey of biomechanical elastography and imaging, from its conceptual inception to clinical applications, ${ }^{64,65}$ was 
presented. Here, we are presenting a brief selective description of existing technologies - that are close to PA imaging technology employing light and sound-for noninvasive and nondestructive elastography imaging (see Sec. 2).

Comparison of various existing elastography imaging modalities is given in Table 1. The existing imaging technologies are limited in certain aspects of clinical applications. Ultrasound imaging modalities primarily rely on the heterogeneity of acoustic impedance [given by the product of bulk modulus $(B)$ and mass density $(\rho)$ of (tissue) specimen] distribution, whereas $\mathrm{CT}$ imaging techniques provide contrast to x-ray attenuation coefficient (electron density). ${ }^{66}$ In the meantime, MRI gives contrast to the magnetic property of tissue (specifically, proton) that is sensitive to water and fat content of the tissue. Meanwhile, research studies have demonstrated that elastography imaging techniques-including ultrasound and MRI ${ }^{13-17}$ - can achieve whole-body (high penetration $\mathrm{cm}$ ) or organ-level imaging with spatial resolutions of the order of hundreds of microns (for ultrasound) ${ }^{67}$ and millimeters (for MRI). ${ }^{68}$ On the other hand, optical coherence elastography (OCE) ${ }^{43-46}$ achieves higher resolution (of the order of $\mu \mathrm{m}^{41,42}$ ) and higher frame rate $(\sim 3,70,000 \text { lines } / s)^{69}$ while the penetration depth is limited to optical transport mean free path $(\sim 1 \mathrm{~mm})$. In a sense, there exists a tradeoff between image resolution and penetration depth; the imaging modalities are constrained to achieve highspatial resolution at the cost of obtainable (higher) imaging depth or vice-versa. In addition, the existing imaging modalities (namely, ultrasound, CT, MRI, OCE, and speckle contrast) for recovery of elastic properties fail to provide physiological information of tissues. On the other hand, photoacoustic (PA) imaging — as a single imaging unit—can provide multiple functional and structural tissue information that is of great clinical interest and value. ${ }^{70}$

PA imaging technology is rapidly emerging and growing since the last decade from laboratory research studies (in late $1990 \mathrm{~s}^{70-72}$ ) to recent clinical trials ${ }^{73}$ for clinical usages as a scanning device. This widespread study around the globe (more than 100 research laboratories dedicating to studies for biomedical applications) is due to its potential and promising features of great biomedical and clinical interest: ${ }^{70,71}$ (1) high-contrast and high-spatial resolution obtainable at penetration depths not achievable with commercially available high-resolution optical-based microscopic imaging modalities (including confocal, two-photon, and OCT); (2) high scalability of imaging, ranging from individual cell to entire body; (3) imaging — with multiple resolution levels - of structural anatomy having contrast in optical absorption coefficient; (4) obtainable pathophysiological information, i.e., physiological states of tissues related to pathological stages through measurement of functional parameters $\left(\mathrm{Hb}, \mathrm{HbO}_{2}, \mathrm{SO}\right.$, total $\left.\mathrm{Hb}\right)$ that control physiological activities, such as metabolism, and molecular and genetic activities; and (5) noninvasive, nondestructive, and nonhazardous (no ionization radiation being employed). The imaging modality has been exploited for various biological (including imaging of single cells and organelles), preclinical (including brain imaging), and clinical (including oncology, ophthalmology, dermatology, gastroenterology, cardiology, epilepsy, and osteoarthritis) studies. Applications of the imaging modality to diagnostic and therapeutic treatments - employing target specific (light absorbing) contrast agents-were also reported. ${ }^{74,75}$ Several studies were reported demonstrating recovery of acoustic property, temperature, and blood flow velocity. ${ }^{76-83}$ Recently, research studies were reported for possible recovery of the elastic property of soft biological tissue with PA imaging technology. ${ }^{84-92}$ This innovative study enhances the horizon of potential applications of PA imaging extending to diagnosis and therapeutic treatment of life-threatening diseases (such as breast and prostate cancer, ${ }^{4}$ brain tumor, ${ }^{93}$ and Alzheimer's diseases ${ }^{94}$ ) that induce contrast in elasticity relative to normal tissues. Currently, PA elastography (PAE) imaging is limited only to qualitative study, and it is in the nurture stage of development. Quantitative assessment (i.e., to quantify measurement) of elasticity and discrimination of contrast in PA signals from elastic and other parameters (including optical absorption coefficient) remain as challenges from technological aspects. Meanwhile, clinical translation of photoacoustic elastography imaging (PAEI) modality is a long way for research studies to accomplish. To our beliefs, these prevailing challenges (including quantitative measurements) may be addressed in near future in a similar fashion that ultrasound elastography (USE) and OCE were enabled to achieve quantitative measurements, i.e., the existing technologies for quantitative recovery of elasticity in USE and OCE may be adapted to PAE as the interrogating (say, optical and acoustic) signals are of similar kind.

This article gives a review of reported studies on PA imaging modalities for recovery, noninvasively and nondestructively, of elastic properties of soft biological tissues. Several review articles on studies of PA imaging modalities and its applications-both for the clinic and basic sciences-were reported in the past..$^{70,71,73,95-106}$ However, to the best of our knowledge, no review on PAE is reported to date. In this article, USE imaging modality is briefly discussed in Sec. 2.1, and Sec. 2.2 brings OCT elastography. We focus on PAE with a detailed account of theory (given in Sec. 2.3).

\section{Elastography Imaging}

Elastography imaging is an art of measuring and subsequent pictorial representation of the (spatial) distribution of tissue stiffness. In preimaging times, medical practitioners routinely assessed tissue-for diagnosis and therapeutic purposes-by subjective (manual) palpation ${ }^{107}$ (as mentioned in Sec. 1). Over the last two decades, elastography has emerged as an active area of research in biomedical imaging that facilitates not only quantitative measurement and clinical usages but also various measures of tissue stiffness (noninvasively and nondestructively). The physical quantity of measurement, in elastography imaging, is broadly carried out in two categories: ${ }^{2}$ (1) quasistatic, in which the derivative of elasticity, i.e., physical parameters depending on the elastic property, is measured; and (2) harmonics, in which measurement of elastic property distribution is achieved.

Fundamentally, elastography imaging consists of two sequential steps. First, the tissue of interest for imaging is subject to external mechanical stimulation and subsequently, the mechanical response of the stressed tissue-either deformation and/or vibration-is evaluated using imaging modalities [ultrasound, ${ }^{11-13} \mathrm{MRl},{ }^{13-17}$ OCT, ${ }^{40-46} \mathrm{CT}$, speckle contrast, ${ }^{51,52}$ and $\left.\mathrm{UMOT}^{59-61}\right]$. The second step is a quantitative characterization of elastic modulus distribution of tissue structure (from measured tissue response to mechanical stimulation) employing an iterative numerical approach that solves a mathematical model to a mechanical problem. ${ }^{9}$ Generally, two methods (namely, static and dynamic) are adopted for the introduction of (external) mechanical stimulation to tissue. In the static method, a constant 


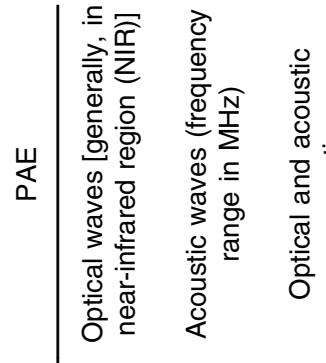

๕.

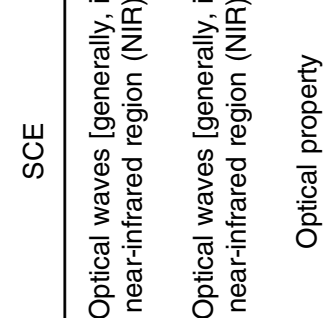

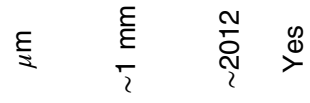

峁

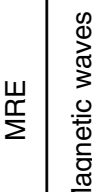



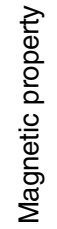

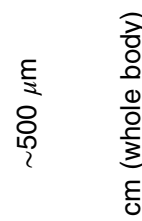

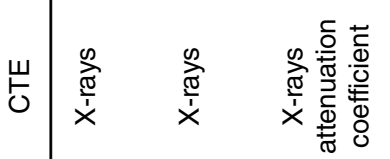



$\cong \widetilde{\widetilde{x}} \quad \cong$

窘高



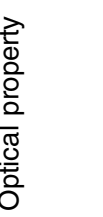

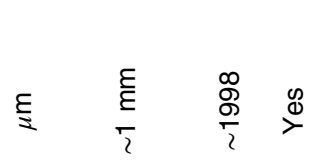


mechanical pressure (not varying with time) is applied to tissue sample surface and the subsequent response of tissue sample to mechanical deformation in the region of interest is evaluated for recovery of elastic property distribution, whereas, in the dynamic method, a time-varying mechanical excitation with frequency close to natural frequency of vibration of tissue $(\mathrm{Hz})$ is induced to the biological sample of interest and the subsequent tissue response, i.e., vibration, is recorded to recover elasticity distribution. For the static method, generally, the tissue is subject to between two plates and then, the elastic modulus of an internal tissue structure having boundaries - detectable by imaging modality - is evaluated from the characterization of tissue deformation (through measurement of the displacement of tissue boundaries) under mechanical stress. While in the dynamic method, a localized and impulsive mechanical strain is induced remotely by an external force (commonly, a focusing ultrasound transducer is employed for inducing remote mechanical excitation $)^{12}$ and reconstruction of elastic property distribution is carried out through evaluation-employing iterative numerical method for solving the governing mechanical problem-of motion pattern induced by externally applied stress, i.e., distribution of elastic property is evaluated through examination of relative motion of tissue elements. ${ }^{108,109}$ Several USE methods-Doppler effect-based velocity measurements, ${ }^{110-112}$ cross-correlation methods ${ }^{113-116}$ to quantify motions (induced internally or externally), visual inspection of $\mathrm{M}$ - and/or Bmodes images, ${ }^{117-119}$ and Fourier-based methods ${ }^{120,121}$ —were reported $^{6}$ and have already been integrated in conventional (clinical) US scanners. Studies for an absolute estimate of tissue stiffness based on the variation of measurement of speeds of wave propagation (faster in stiffer tissues) were also reported. ${ }^{122,123}$ More recently, MR elastography has provided evidence of its efficacy in tissue characterization and the imaging modality is increasingly incorporated in clinical practice.

\subsection{Ultrasound Elastography Imaging}

USE is an imaging technology for recovery of (spatial) distribution of tissue mechanical properties. The technology was first introduced in the early 1990s. ${ }^{6,124,125}$ Since then, from technological aspects, it has been evolving continuously, and it has further been developed and refined in recent years so as to enable quantitative assessments of tissue stiffness. USE has been explored for various clinical applications in recent years, and it has been introduced into a clinical routine for specific applications including liver fibrosis and breast cancers. While USE has shown promising results for noninvasive assessment of liver fibrosis, the technology has been emerging for applications in breast, thyroid, prostate, kidney, and lymph node imaging. ${ }^{125}$

Figure 1 gives a representative classification tree of various existing USE imaging modalities. In general, USE imaging technology can be classified into three main subgroups: ${ }^{125}$ (1) strain imaging methods, which is generally known as compression (or compressional) elastography, ${ }^{6}{ }^{(2)}$ shear wave elastography (SWE), and (3) B-mode elastography. In strain or compressional imaging, mechanical strain $(\varepsilon)$-which is induced along the direction of a mechanical stress $(\sigma)$ being applied externally (normal to tissue surface) - is measured experimentally and subsequently, Young's modulus $(E)$ is estimated using stress-strain relationship given by Hooke's law $[\sigma=\Gamma \varepsilon$, where $\Gamma$ is the constant coefficient called elastic modulus [Young's modulus $(E)$ for linear strain; bulk modulus $(B)$ for volume strain; and shear modulus $(G)$ for tangential strain], and $\varepsilon$ is measured strain in response to an applied mechanical stress $(\sigma)$ ] within elastic limit. This elastic modulus $(\Gamma)$ —which relates mechanical (static) deformation $(\varepsilon)$ to restoring stress $(\sigma)$ - characterizes propagation of mechanical waves in a (tissue) medium, i.e., speed and frequency of mechanical waves. Ultrasound shear wave imaging measures, experimentally, speed $\left(v_{S}\right)$ of propagation of (transverse) shear waves and, in turn, Young's modulus $(E)$ is obtained from measured $v_{S}$ using the relationships of $G$, $E$, and $v_{S}$ [given by $v_{S}=\sqrt{\frac{G}{\rho}}$ and $E=2(\gamma+1) G$, where $G$ is shear modulus, $\gamma$ is Poisson's ratio $\left(\sim 0.5\right.$ for soft tissue $\left.{ }^{125}\right]$, and $\rho$ is mass density). While, in B-mode imaging, the speed of propagation of longitudinal wave $\left(v_{L}\right)$ is measured experimentally and then, bulk modulus $(B)$ of sample material is recovered using $v_{L}=\sqrt{\frac{B}{\rho}}$. Shortly, depending on mechanisms of inducing external mechanical stimulation and the subsequent measurement of tissue response to stimulation, different mechanical properties (elastic moduli) of the sample material (namely, Young's, shear, and bulk) can be obtained. The three types of elastic moduli-associated with three types of mechanical deformation or strain - are not independent to each other, but they are closely related (as solid objects attempt to conserve original volume), which is dictated by Poisson's ratio $(\gamma)$. The speed of propagation of longitudinal waves - as it is measured experimentally in B-mode USE-gives a relatively low dependence on mechanical (bulk modulus) properties and, hence, measured bulk modulus $(B)$ provides a low contrast to the characterization of (soft) tissue pathological stages in elastography measurements. However, low speed of propagation of shear waves in soft tissues gives a high contrast in the measurement of $G,{ }^{125}$ which results in facilitating a sensitive way to characterize pathological stages of soft tissues associated with above mentioned life-threatening diseases.

Depending on an externally induced mechanical stimulation and approach to study tissue response to stimulation, strain imaging is further subclassified as strain elastography imaging and acoustic radiation force impulse (ARFI) strain imaging while, for shear imaging, it is subclassified as point shear wave elastography (pSWE) imaging, 2-D SWE, and 1-D transient elastography. In contrast to SE imaging [where a strain (static or dynamic) is applied remotely to tissue region of interest], in ARFI strain imaging technique, a high-intensity acoustic pulse wave of power $\left[\sim 1400 \mathrm{~W} / \mathrm{cm}^{2}\right.$ (spatial peak pulse average) and $\sim 0.7 \mathrm{~W} / \mathrm{cm}^{2}$ (spatial peak temporal average)] and of pulse duration ( $\sim 0.1$ to $0.5 \mathrm{~ms}$ ) is used to induce tissue mechanical stimulation (displacement of $\sim 10$ to $20 \mu \mathrm{m}$ ) over a narrow prespecified focal region (of focusing transducer) deep inside the body. These high-intensity focusing acoustic waves are introduced, remotely, to a tissue sample in the normal direction (i.e., perpendicular) to the tissue surface. Again, in comparison of strain imaging and shear wave imaging, tissue displacement that is induced along the direction of applied normal stress is measured (in strain imaging), whereas, in SWE, shear wavesthat propagate in a direction perpendicular to the vibratory motion of constituent particles of tissue sample - are measured. In pSWE, ARFI is used to induce tissue displacement at a single prespecified (focal) location similar to ARFI strain imaging. Then, the speed $\left(v_{S}\right)$ of shear waves propagation, which is perpendicular to the plane of excitation, is measured either directly or converted to Young's modulus (E). In 2-D SWE, instead of a single focal location as in ARFI strain imaging and pSWE, multiple focal zones are induced in rapid succession, 


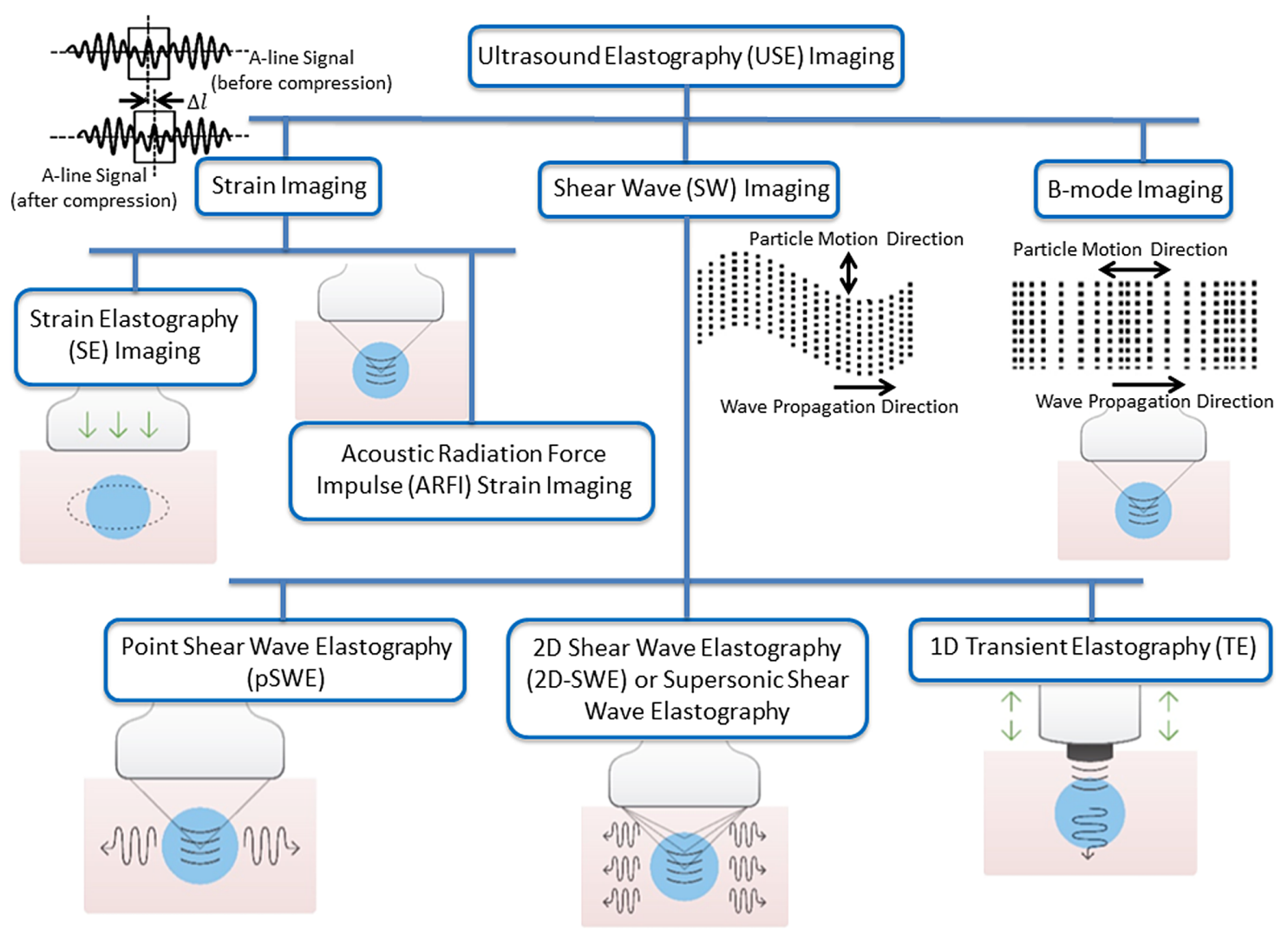

Fig. 1 Classification tree of various USE imaging methods. In strain imaging, to estimate mechanical properties [Young's modulus $(E)$ ], tissue displacement is measured by correlation of radio-frequency (RF) echo signals over a narrow prespecified search windows (boxes) in states before and after compression. While, in shear wave imaging, speed $\left(v_{S}\right)$ of shear wave propagation-associated with particle motion and perpendicular to direction of wave propagation -is measured and, in turn, shear modulus $(G)$ is estimated. In B-mode imaging, particle motion parallel to direction of wave propagation is characterized to obtain speed $\left(v_{L}\right)$ of longitudinal wave propagation and hence, bulk modulus $(B)$. ARFI technique is employed-in all of subgroup methods except for SE imaging and 1-D transient elastography (TE) imaging - to induce mechanical excitation of deep-seated tissue remotely. In SE imaging, tissue surface is compressed; in TE imaging, a conventional US probe is employed to induce mechanical excitation.

faster than shear wave speed whereby creating a nearly cylindrical shear wave cone. This cone beam-like mechanical stimulation facilitates real-time monitoring of shear waves in 2-D for measurement of shear wave speed or Young's modulus $(E)$. Again, in 1-D TE imaging, a single ultrasound probe serves both the purpose of inducing mechanical stimulation as well as ultrasound detection in pulse-echo mode, i.e., the ultrasound probe, first, serves as an external mechanical vibrating device exerting a controlled vibrating "punch" on body surface to generate shear waves (which propagate through tissue) and the same probe is served, in a time-sharing manner, to detect time-resolve (A-mode) US signal from which the speed of shear wave propagation and Young's modulus $(E)$ is estimated.

\subsection{Optical Coherence Elastography Imaging}

A few years later, the advent of USE (in 1991), by the end of the 1990s, OCT was exploited for elastography imaging and its studies. ${ }^{22}$ In fact, from historical aspects, even though scientific venture or inception of OCT-based elastography dates back close to three decades, it is only in these past few years (say, since 2012 or so) that rapid progress in OCE was witnessed. With reference to earlier reported works, abundantly cited in numerous reviews, several variants of methods and techniques-like compressional OCE that was abandoned for a while-have demonstrated excellent results and feasibility for practical (clinical) applications. In this regard, reported research ${ }^{40,126-129}$ played a pioneering and stimulating role in OCE. As a fruit of continuous efforts, presently, the challenges of facilitating only relative measurements of stiffness or elasticity are successfully addressed; thereby, quantitative mapping of strain and Young's modulus at high-resolution is achievable. Furthermore, an estimate of a single small-strain value of Young's modulus, obtaining from nonlinear stress-strain curves, has also been successfully demonstrated by several groups. Several dynamic methods, ${ }^{33}$ which differ significantly from previously discussed variants, came into existence. Compressional OCE, which was originally proposed (in 1998) by Schmitt, ${ }^{22}$ has been adopted by several groups in recent years and there have been significant advances in aspects of signal processing and analysis. In contrast to techniques initially proposed as well as tested in numerous reported works, ${ }^{130}$ it was demonstrated that correlation techniques ${ }^{23}$ for mapping of strain, which is fundamentally based on analysis of phasesensitivity of OCT signals, enhance obtainable contrast and sensitivity. ${ }^{24-26}$ Different methods of quantitative mapping of stiffness, which is based on the utilization of translucent reference layers, were presented in Refs. 27 and 28. Clinical 
applications-for oncology- ${ }^{29-31}$ and for ophthalmology ${ }^{32-34}$ of phase-sensitive strain and stiffness mapping were reported in the past. Obtaining of nonlinear curves was demonstrated and discussed in detail in Refs. 35 and 36. Several review papers, in which significant attention was paid to various dynamic OCE methods, were reported. ${ }^{37-39}$ References 22 and 40 give a detailed study of OCE demonstrating that OCE can generate a microscale optical mapping of biomechanical properties of tissues.

In OCE, in contrast to USE, responses of tissue material to externally applied mechanical stresses (static or dynamics) are interrogated through interferometric-based optical technique. ${ }^{22}$ Quantitative measurements of elastic moduli-experiments being performed in tissue-mimicking phantoms and ex vivo human tissues-were reported in the past. The study ${ }^{40}$ showed the mapping of dynamic elastic moduli of normal and neoplastic ex vivo human breast tissue with an achievable sensitivity of measurements $(\sim 0.08 \%)$. In the study, a dynamic OCE method-in which tissue sample of interest is excited by mechanical waves, and biomechanical properties are estimated by solving wave equations in contrast to computationally expensive cross-correlation techniques-was employed. The technique addresses the difficulty of conventional OCE to provide quantitative elastography based on cross-correlation of speckles on microscale. In conventional OCE technology, ${ }^{126,127}$ phaseresolved measurement methods were used and are successful for providing intravascular imaging, ${ }^{128,129}$ atherosclerotic tissue imaging, ${ }^{130}$ and imaging of engineered and developing tissues. $^{131}$

\subsection{Photoacoustic Elastography Imaging}

Reported studies on photoacoustic elastography imaging (PAEI) are very limited. To the best of our knowledge, it was only in 2011 that study on the recovery of viscoelasticity of soft tissues using PA imaging modality was reported. ${ }^{84}$ In this study, a phase-resolved technique was employed. Singh and Jiang ${ }^{85}$ reported an experimental study that demonstrates attribution of elastic properties of light absorbing targets to the generation of ultrasound signals induced due to PA effect, i.e., generation of acoustic waves upon illumination of a sample material by a short duration (ns) pulse optical beam. The study investigated variation in the strength of PA signals for various targets, with different elastic coefficients and various sizes, embedded in a background phantom. A nondestructive method-which is based on the characterization of the resonance frequency of PA signal, i.e., characterization of maximum amplitude of acoustic (PA) waves with a the variation of operating frequency of acoustic sensor-for determining Young's modulus of tumors was proposed. ${ }^{86}$ In this study, a miniaturized acoustic sensor was developed and the results demonstrate the feasibility of PA technology to mechanically characterize soft tissue by diagnostic endoscopy. Zhao et al. ${ }^{87}$ report a simultaneous recovery of optical absorption coefficient (and hence, the structure of biological tissue) and viscoelasticity of soft tissues. In the proposed technique, structural information is obtained from the amplitude of PA signals while elastic property measurement is recovered from phase-shift of PA signals-that are resulted due to damping effect of soft tissue-relative to pulse optical signal. Multispectral technique-for recovery of elasticity employing PA tomography imaging modality — was reported with the study being carried out in experiments and simulation. ${ }^{88}$ In 2017, a reported study ${ }^{89}$ demonstrated selection in operating frequency of US transducer being employed for boundary detection of PA signals that is resulted due to contrast in elasticity distribution in sample material other than that of optical absorption coefficient, i.e., for detecting PA signals due to contrast in mechanical elasticity, a US transducer of higher operating frequency $(\sim 50 \mathrm{MHz})$ is demanded. Shear wave-based PA imaging modality was reported for quantitative recovery of mechanical properties of soft tissues both elasticity and viscosity - measuring rise-time of thermoelastic displacement and biomedical application of the approach-were validated in ex-vivo studies for liver cirrhosis detection. ${ }^{90}$ Pengfei et al. ${ }^{91}$ developed PA tomography system for imaging elasticity (Young's modulus) through the study of dynamics of microparticle embedded in sample material while the same group reported their study on characterization of blood vessel elasticity for end application targeted to acute myocardial infarction and stroke, and human skeleton muscle. ${ }^{92}$

\subsubsection{Theory}

PA imaging is, fundamentally, based on PA effect, which is the generation of mechanical (sound) waves in a sample material associated with illumination of electromagnetic (EM) radiation for a short duration, ${ }^{132-134}$ i.e., it is a phenomenon of the generation of acoustic waves due to transient absorption of EM energy and the subsequent rapid heating in a material sample irradiated with EM waves. ${ }^{85}$ The entire process of the effect can be subgrouped into three distinct stages: (1) transient irradiation of tissue sample of interest with EM (light) waves, (2) generation of mechanical (acoustic) waves in a wide range of frequency $(\mathrm{Hz}$ to $\mathrm{GHz}),{ }^{132,135}$ and (3) propagation of PA waves.

In the transient illumination stage, a narrow pulse of EM waves (pulse-width of a few nanoseconds) with operating wavelengths in visible or near-infrared (NIR) region is delivered to the tissue surface to irradiate a prespecified region-of-interest (RoI) at a certain depth (submillimeters to a few centimeters). This stage is governed by Maxwell's theory of EM radiation that governs the propagation of EM waves in a (scattering or nonscattering) medium. ${ }^{136}$ In other words, the propagation stage is characterized by the distribution of physical parameters-electric permittivity $[\epsilon(\vec{r})]$ and magnetic permeability $[\mu(\vec{r})]$ and, in turn, optical properties [absorption $\left[\mu_{a}(\vec{r})\right]$ and scattering $\left[\mu_{s}(\vec{r})\right]$ coefficients, and refractive index $[n(\vec{r})]$-in propagating medium (tissues, in our case) and characteristics of the illuminating radiation [operating wavelength $(\lambda)$ and radiation energy $(E)$. As EM (light) waves are propagating through tissue medium, optical energy is deposited in tissue that is characterized by $\mu_{a}(\vec{r})$ and $\mu_{s}(\vec{r})$. This absorbed energy is converted into heat energy through vibrational and oscillational relaxation of constituent particles/molecules of the medium, therefore resulting in a localized rise of temperature over the radiation-irradiated tissue region. ${ }^{85}$ For a given intensity of the incident optical (laser) beam, initial rise of temperature $[\Delta T(\vec{r})]$ is dependent on $\mu_{a}(\vec{r})$. Due to rapid heating, associated with transient illumination of radiation under the constraint of illumination duration to be less than time scales of thermal and stress confinements, irradiated tissue undergoes thermoelastic expansion ${ }^{99}$ inducing a transient rise in pressure. This initial pressure rise is commonly called PA pressure. Shortly, generation of PA waves is fundamentally based on PA effect, ${ }^{134}$ which is the generation of acoustic waves-due to thermoelastic expansion-upon transient irradiation (short duration of a few nanoseconds) of sample 
material with a light beam. In similar fashion, in thermoacoustic (TA) effect, which is exploited in TA imaging, short-pulse microwaves irradiating a sample induce transient heating due to absorption of EM energy that is in the microwave frequency range. ${ }^{135}$ In both cases, this transient heating-due to absorption of transient EM waves-produces a fractional change in volume $(V)$. From thermodynamics, the fractional volume change $(\Delta V)$ is given by ${ }^{137,138}$

$\Delta V=-\kappa \Delta P+\beta \Delta T$,

where $\Delta P$ represents pressure change (or initial pressure rise), $\Delta T$ represents temperature change, $\kappa$ represents isothermal compressibility, and $\beta$ represents the thermal coefficient of expansion. When temporal pulse-width of irradiating EM waves is less than the time-scale of thermal and acoustic confinements in the medium, $\Delta V / V$ [in Eq. (1)] can be neglected. ${ }^{135}$ Again, neglecting nonthermal effects (like fluorescence) and assuming irradiating EM energy is completely converted to thermal energy giving rise to a raise in $T$, initial temperature rise $(\Delta T(\vec{r}))$ is obtained as follows: ${ }^{85,139}$

$\Delta T(\vec{r})=\frac{1}{\left.\rho(\vec{r}) C_{V}(\vec{r})\right)} \mu_{a}(\vec{r}) \phi\left[\vec{r}, \mu_{a}(\vec{r}), \mu_{a}(\vec{r}), g\right]$,

where $\mu_{a}(\vec{r})$ is intrinsic absorption coefficient; $\phi\left[\vec{r}, \mu_{a}(\vec{r})\right.$, $\left.\mu_{a}(\vec{r}), g\right]$ is the spatial distribution of optical fluence depending on $\mu_{a}(\vec{r}), \mu_{a}(\vec{r})$, and $g ; g$ is anisotropy factor; and $C_{V}(\vec{r})$ is specific heat capacity at constant volume.

If $P_{0}(\vec{r})$ is initial pressure rise, i.e., initial PA waves, considering residual pressure prior to light illumination as a reference, one can write as follows: ${ }^{85,139}$

$P_{0}(\vec{r})=\Gamma(\vec{r}) A_{e}(\vec{r})$,

where $\Gamma(\vec{r})=\frac{\beta(\vec{r}) v_{L}^{2}(\vec{r})}{C_{p}}$ represents Grüneisen parameter; $C_{p}$ is specific heat capacity at constant pressure; $\beta(\vec{r})$ is thermal expansion coefficient; and $A_{e}\left[=\mu_{a}(\vec{r}) \phi(\vec{r})\right]$ is specific optical absorption. Equation (3) shows that initial pressure rise, $P_{0}(\vec{r})$, depends on thermal expansion and hence, initial PA pressure is primarily a strain source. By strain source, it means that strength of strain pulse (i.e., PA source)—which subsequently propagates in surrounding medium-is characterized by a variation in the elastic modulus of the target (i.e., a specific region of interest) relative to the background region. For example, if elastic modulus of the target is lower in comparison to that of the background, the induced strain is lower while in the case of the higher elastic modulus of the target with respect to that of surrounding medium, strain pulse and associated PA signals are comparatively higher. Shortly, light-induced strain pulses or PA signals are dependent on elastic property distribution. In an elastic medium like soft tissues, a change in spatially and temporally varying pressure, $P_{0}(\vec{r}, t)$, initiates mechanical (acoustic) waves ${ }^{139}$ - initially localized at laser irradiating points-that have a tendency to propagate in elastic medium through interaction or under the influence of neighboring constituent particles/molecules that undergo constrained oscillatory motions about thermal equilibrium positions, i.e., acoustic waves are propagated while transferring momentum. ${ }^{51,140}$ Propagation of PA pressure wave in an elastic medium is governed by a second-order partial differential equation as follows: $:^{141,142}$ $\nabla^{2} P-\frac{1}{v_{L}^{2}} \frac{\partial^{2} P}{\partial t^{2}}=\frac{-\beta}{C_{p}} \frac{\partial H}{\partial t}$

where $v_{L}$ is the speed of sound in the medium, $C_{p}$ is specific heat at constant pressure, and $H$ is heating function given by thermal heat energy per unit time $(t)$.

In PA imaging, acoustic signals with frequencies of order of $\mathrm{MHz}$ (in the case for elastography, $\sim 50 \mathrm{MHz}$ ) are selectively picked up keeping an acoustic sensor (commonly, the ultrasound transducer is used) — that may consist of single element or an array of ultrasound transducer elements - at boundary of sample of interest. Several techniques-including model-based iterative methods that involve solving of differential equation [Eq. (4)] and beam-forming methods-are reported for reconstruction of initial pressure rise $\left[P_{0}(\vec{r})\right]$ from boundary measurement of $[P(\vec{r})]$ being carried out broadly in various ways: (1) tomography-based techniques, which either involve solving differential equation [Eq. (4)] with model-based iterative methods or beam-forming methods, and (2) microscopy techniques, where a tightly focusing ultrasound transducer is employed to detect initial PA signals over the narrow focal region. The detail of PA microscopy is presented in Sec. 2.3.3. Tissue material properties are derived from measured initial pressure signals. Several research studies are reported in the literature on the derivation of $\mu_{a}(\vec{r}), v_{L}(\vec{r})$, and $T(\vec{r})$ from measured PA signals in sample boundary. Our previous experimental study ${ }^{85}$ - underscored herein in Sec. 2.3.3-demonstrated that elastic coefficient distribution $[E(\vec{r})]$ of a sample material attributes to the generation of PA waves, i.e., strength of PA signal is dependent on the stiffness of sample material that supports propagation of mechanical acoustic waves through it. Earlier, it was understood that the generation of PA waves is only due to $\mu_{a}(\vec{r}) \cdot{ }^{132-134,143}$ Experimental results-in our studydemonstrated the possibility of recovering elastic property of tissues using PA-imaging technique. Our study extends the horizon of PA-imaging technology for diagnosis, assessment, and therapeutic treatments of various life-threatening diseases, which are characterized with a change in the elastic property of tissues with pathological stages. ${ }^{4,93,94}$

\subsubsection{Frequency selection of ultrasound sensor for detection of PA-signal contrast due to elastic coefficient}

Equation (3) shows that-for a given intensity of incident EM radiation- $P_{0}(\vec{r})$ is characterized by $\mu_{a}(\vec{r})$ as well as $\beta(\vec{r})$, whereas $\beta(\vec{r})$ is a characteristic of elastic property $[E(\vec{r})]$ that possesses distinctive frequency response to an externally induced mechanical stimulation. This initial pressure raise gives rise to a generation of acoustic waves with a frequency of the order of $\mathrm{Hz}$ to GHz. Studies ${ }^{85,134,135,139}$ showed that frequency response of generated acoustic signals is characterized by associated contrast mechanisms. Distribution of concentration of water and ions is the main source of contrast in acoustic waves with frequency $\sim \mathrm{GHz}$ in association to an irradiation with EM waves in microwave range-called TA waves-whereas constituents of blood $\left(\mathrm{Hb}, \mathrm{HbO}_{2}\right.$, total $\mathrm{Hb}$, and oxygen saturation) and melanin are the major sources of acoustic waves with frequency $\mathrm{MHz}$ in response to an irradiation with EM waves in visible or near-infrared region-known as PA waves. ${ }^{135}$ This indicates that one needs to adapt different types of sources and acoustic sensing systems of different central frequencies 
for measurement and recovery of various components of tissue optical absorption that are of clinical importance for diagnosis and therapeutic treatments. Say, in TA-imaging, microwaves (frequency of a few $\mathrm{GHz}$ and pulse-width of a few microseconds) are used as source and antenna-based microwave sensors are used as signal sensing systems. ${ }^{135}$ In PA-imaging, shortpulse laser with a pulse width of a few nanoseconds is used as a source and US-transducer is used as a detector. Generally, US transducers $(\sim 1$ to $10 \mathrm{MHz})$ were employed for recovery of $\mu_{a}(\vec{r}) \cdot{ }^{132,134,143}$ We observed that (experimental results are shown later), in a medium with contrast in spatial distribution of elastic property while maintaining uniform distribution in $\mu_{a}$, US transducers (with operating frequency $\sim 1$ to $10 \mathrm{MHz}$ ) are not sensitive to detect light-induced PA signal contrast, and it is detectable with US transducer $\sim 50 \mathrm{MHz}$, i.e., the dominant frequency of PA-signals is $\sim 50 \mathrm{MHz}$. In other words, the frequency response of light-induced PA-signals is shifted from $\sim 1$ to $10 \mathrm{MHz}$ [in the case of contrast in $\mu_{a}(\vec{r})$ ] to $\sim 50 \mathrm{MHz}$ [in the case of contrast in $E(\vec{r})]$. From Classical Physics, in an elastic system consisting of distribution of mass that can be considered as a system of different masses coupled to each other by springs of different spring constants, possible frequencies of natural mode of vibration of the masses are increased in comparison to natural frequencies of vibration of individual masses when they are left uncoupled. ${ }^{144}$ This physical system of interconnected springs has a close similarity to a (tissue sample) system of clinical interest that is constituted by biomolecules (including organic) of diverse sizes and shapes, and intermolecular distances. A proper coupling in propagation of mechanical (PA) waves, being facilitated by natural frequency of vibration of a given coupled-mechanical system (biological tissue, in this case), may be attributed by distribution of physical entities (including biomolecules and inhomogeneity) with an intermolecular distance close to acoustic wavelength corresponding to acoustic frequency of interest $(\mathrm{MHz}$, in the case of $\mathrm{PA}$ imaging).

In our previous report, ${ }^{85}$ we presented an experimental study on the selection of frequency response of PA signals that are generated due to contrast in distributing elastic property of the sample material. We observed that response is biased with maximal peak response at a particular operating frequency of the acoustic sensor, and consequently, detection of PA signals demands a selection in operating frequency of US transducer being employed as an acoustic sensor.

\subsubsection{Photoacoustic microscopy elastography imaging}

Figure 2 depicts a schematic diagram of PA microscopy (PAM) imaging system, more specifically acoustic-resolution (AR) PAM system, which we employed as an imaging unit in the study of PAM elastography. ${ }^{85,89}$ In PAM imaging modality, a highly energetic optical beam is adapted to illuminate tissue sample of interest over a prespecified region (for a short duration $\mathrm{ns})$, and the resulted initial pressure rise $\left[P_{0}\right.$, given by Eq. (3) $]$ is selectively picked-up by employing a tightly focusing ultrasound transducer. In the sense, tightly focusing nature of an ultrasound transducer enables to detect acoustic signal over a narrow region specified by its focal zone (focal spot size $\sim 60 \mu \mathrm{m})$. In this way, PAM enables to pick-up initial pressure $\left(P_{0}\right)$, in contrast to photoacoustic tomography (PAT), where (secondary) pressure waves $(P)$-given by Eq. (4)-are acquired by keeping the unfocused acoustic sensor in sample boundary. A detail description of PAT elastography imaging,



Fig. 2 Schematic diagram of AR-PAM imaging system.

from experimental aspects, is provided in Sec. 2.3.4. Note that both initial pressure rise $\left(P_{0}\right)$ and secondary pressure waves $(P)$ propagating in an elastic medium provide vital tissue thermal, mechanical, acoustic, and optical properties (as explained in Sec. 2.3.1). From Eqs. (3) and (4), $P_{0}$ and $P$ are characteristics of acoustic velocity in the propagating medium and hence, bulkmodulus $\left(B=\rho / v_{L}^{2}\right)$. However, elastic parameters (bulk, shear, and Young's moduli) of the medium are not independent to one another but they are inter-related by Poisson's ratio [say, Young's modulus $E=3(1-2 \gamma) B,{ }^{9}$ where $\gamma$ is Poisson's ratio]. In the sense, PA signals $\left(P_{0}\right.$ and $\left.P\right)$ are sensitive to a variation of Young's modulus $(E)$. Briefly, to describe our AR-PAM, the imaging system comprises two main subunits, i.e., optical pulse illumination and acoustic detection. In illumination, a beam of coherent light (pulse-width $\sim 6 \mathrm{~ns}$, pulse repetition frequency $\sim 10 \mathrm{~Hz}$, wavelength $\sim 720 \mathrm{~nm}$, and energy density $\sim 22 \mathrm{~mJ} /$ $\mathrm{cm}^{2}$ ) from a laser source (Surelite OPO Plus, Continuum) was delivered-through an assembly of two optical fibers (as shown in Fig. 2) - to irradiate prespecified RoI of tissuemimicking (agar) phantom. For picking-up pulse laser-induced PA-signals-selectively, from a narrow focal region of the transducer-a tightly focusing ultrasound transducer was kept in sample boundary. In the experimental studies, various focusing ultrasound transducers-of different operating central frequencies $(1,3.5,7$, and $50 \mathrm{MHz}$ )—were employed. The transducer ( $\sim 50 \mathrm{MHz}$ ) was custom-make (focal spot size $\sim 61 \mu \mathrm{m}$, focal length $5 \mathrm{~mm}$, and length of the focal zone $\sim 1 \mathrm{~mm}$ ), whereas the other transducers (focal length $\sim 10 \mathrm{~mm}$ ) were supplied by Panametrics, Olympus Corp. During experiments, optical fibers and transducer-which are housed together in a holding system -were immersed inside an acoustic-coupling medium (water, in our case) in a container for proper coupling of ultrasound transmission. The sample was kept in a fixed position below the water container. It is to note that an acoustic coupling gel was applied over the sample surface for proper coupling of ultrasound transmission. A 3-D image is achieved by raster scanning technique whereby time-resolved measurement at a scanning position gives a 1-D image data. Various focusing transducers with operating frequencies $(1,3.5,7$, and $50 \mathrm{MHz})$ were employed for the study of the frequency response of PA signals with signal contrast from the elastic property. ${ }^{89}$ In the studies, agar-based tissue-mimicking phantoms-whose physical properties [mechanical and optical (scattering and absorption)] can be tailored independently ${ }^{145-147}$ - were employed as investigating (imaging) samples. 
Experimental studies were reported ${ }^{85}$ for investigation of variation in strength of (short pulse) laser-induced PA signals with elastic coefficient of targets and, in turn, for studying shift in frequency response of ultrasound-detecting transducer, i.e., change in natural frequency of mechanical vibration of tissue targets, with change in contrast of either optical or mechanical properties of targets. Experimental studies were performed in agar-based tissue-mimicking phantoms-with targets (y's) of various contrasts in optical and mechanical properties [see Fig. 3(a)] and in sizes [Fig. 3(b)] being embedded in a background sample-simulating tissue abnormalities (due to diseases) in normal background tissues. Moreover, in our previously reported studies, ${ }^{89}$ experiments were also carried out-adopting third tissue-mimicking phantom [shown in Fig. 3(c)] - to demonstrate and validate variation in the strength of laser-induced PA-signal with a selection of the operating frequency of US transducer, which is used as an acoustic (signal) sensor, for various types of contrasts $\left(\mu_{a}\right.$ and $E$ ) of targets. To describe briefly, for all targets $\left[T_{1}\right.$ to $T_{6}$, in Fig. 3(a)], $\mu_{a}$ and $\mu_{s}$ were fixed similar to that of background $\left(0.01\right.$ and $1.20 \mathrm{~mm}^{-1}$, respectively) except for the second target $\left(T_{2}\right)$ in which $\mu_{a}=0.02 \mathrm{~mm}^{-1}$. Against $E=94 \mathrm{kPa}$ (for background), elastic coefficients [Young's modulus $(E)$ ] for targets $\left(T_{3}\right.$ to $\left.T_{6}\right)$ were tailored to $345,269,202$, and $143 \mathrm{kPa}$, respectively, which were achieved by variation of agar concentration with $2 \mathrm{mg}$ (background, $T_{1}$, and $\left.T_{2}\right), 4.0 \mathrm{mg}\left(T_{3}\right), 3.5 \mathrm{mg}\left(T_{4}\right), 3.0 \mathrm{mg}\left(T_{5}\right)$, and $2.5 \mathrm{mg}\left(T_{6}\right)$ to $100 \mathrm{~mL}$ of water. A detailed description of (agar) sample preparation is provided in Ref. 89. These elastic properties are found to be within the range of elastic property of (cancerous) soft tissues. ${ }^{9,145}$ As shown in Fig. 3(b), targets of different cross-sections $\left[0.5 \times 0.5 \mathrm{~mm}^{2}, 1.0 \times 0.5 \mathrm{~mm}^{2}\right.$, $2.0 \times 0.5 \mathrm{~mm}^{2}$, and $3.0 \times 0.5 \mathrm{~mm}^{2}(x \times z)$ for $T_{1}$ to $\left.T_{4}\right]$ were embedded in background phantom for studying the dependence of PA signals on target size while optical and elastic properties of embedded targets were tailored similarly to those of background $(E=94 \mathrm{kPa})$. In the third sample [Fig. 3(c)], two strips of target $\left(t_{1}\right.$ and $t_{2}$ with rectangular cross-section $\left[2.0 \times 0.5 \mathrm{~mm}^{2}\right.$ in $x z$-plane $\left.)\right]$-having contrast in elastic coefficient [Young's modulus, $E=202 \mathrm{kPa}$ (with no contrast in $\mu_{a}$, for $t_{1}$ ) while having contrast in $\mu_{a}=0.02 \mathrm{~mm}^{-1}$ (with no contrast in mechanical properties, for $t_{2}$ )]—were embedded in background phantom.
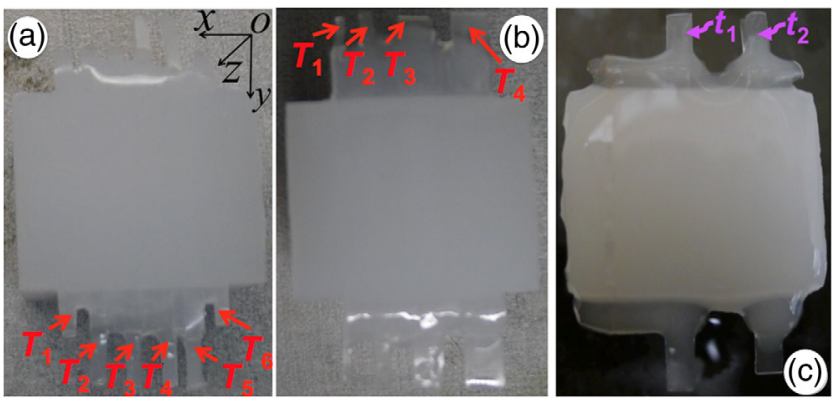

Fig. 3 (a) Imaging targets $\left(T_{1}\right.$ to $T_{6}$ ) of similar cross-sections $(1.0 \mathrm{~mm} \times 0.5 \mathrm{~mm}$ in $x z$-plane) with variation in elastic coefficient were embedded in background phantom $\left[25 \times 20 \times 20 \mathrm{~mm}^{3}\right.$ $(x \times y \times z)]$. (b) Targets of different cross-sections $(0.5 \mathrm{~mm} \times 0.5 \mathrm{~mm}$ to $3.0 \mathrm{~mm} \times 0.5 \mathrm{~mm}$ for $T_{1}$ to $T_{4}$ in $x z$-plane) with optical and mechanical properties similar to that of background phantom. (c) Contrast in $E$ (with similar $\mu_{a}$ for $t_{1}$ ) and $\mu_{a}$ (with similar $E$ for $t_{2}$ ) with respect to that of background sample.
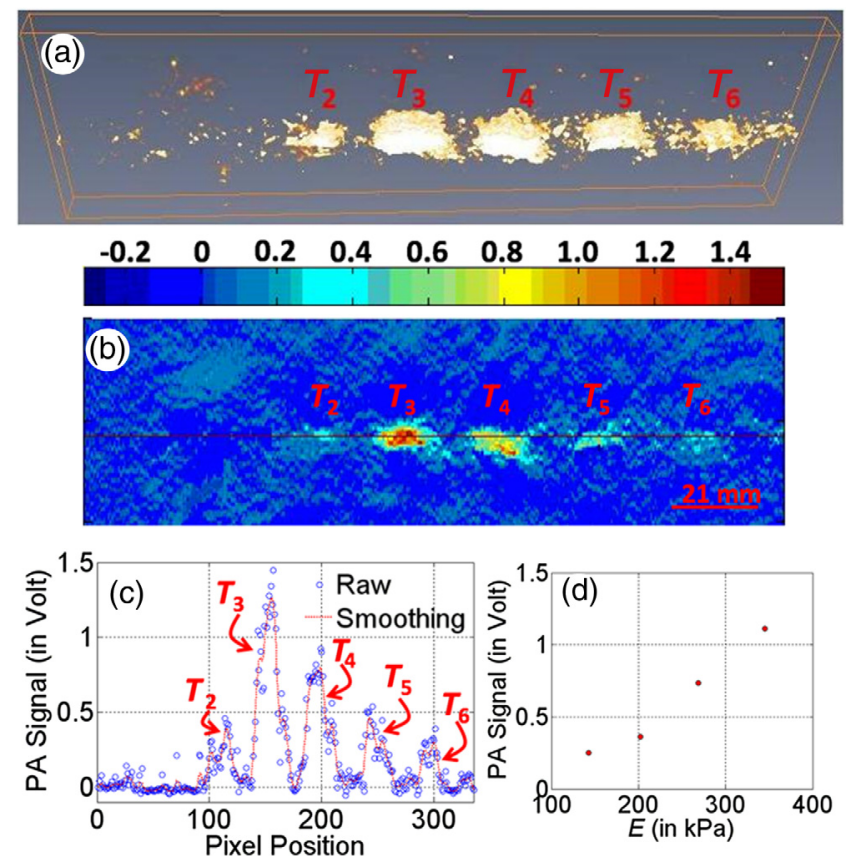

Fig. 4 3-D reconstructed image (a) of PA signals obtained from a sequence of 2-D PA-representative images (b) by employing Amira software. (c) Line plot showing the variation of PA signals along the line as marked in (a). (d) Variation of PA signals (as obtained from target regions) with Young's modulus, E. Experiments were performed in sample depicted in Fig. 3(a) while a tightly focusing transducer of operating frequency $50 \mathrm{MHz}$ was employed as acoustic sensor.

Figure 4(a) depicts 3-D reconstructed image, which is representative of detected laser-induced PA signals obtained through extending 1-D scanning (along the $x$ axis) toward $y$ axis, and Fig. 4(b) gives a 2-D reconstructed image that is representative to PA signals obtained through 1-D scanning of the sample [shown in Fig. 3(a)] along the $x$ axis in the middle portion of the sample. In these experiments, the focusing transducer of the operating frequency $50 \mathrm{MHz}$ was employed as an acoustic sensor. The image demonstrates that detected PA signals have contrast for targets $\left(T_{2}\right.$ to $\left.T_{6}\right)$ with respect to the background while target $\left(T_{1}\right)$ is not detectable, i.e., $T_{1}$ gives no PA signal contrast. Figure 4(c) gives line-plot showing a variation of PA-signals for all pixels along a marked line [as indicated in Fig. 4(b)]. Observed contrast in PA signals, corresponding to targets $\left(T_{3}\right.$ to $\left.T_{6}\right)$, is due to the contrast in elastic coefficient (Young's modulus, $E$ ) of embedded targets (which were tailored to have contrast in the elastic property only while maintaining similar optical properties, i.e., $\mu_{a}$ and $\mu_{s}$ ) in comparison to that of background phantom. Observed PA-signal contrast corresponding to target $\left(T_{2}\right)$, whose elastic coefficient $(E)$ was tailored similarly to that of background phantom while contrast being maintained only in $\mu_{a}$, is because of contrast in optical absorption coefficient $\left(\mu_{a}\right)$. Because, for the target $\left(T_{1}\right)$ that was tailored with no contrast both in optical and elastic coefficients with respect to background, there is no contrast observable in the reconstructed PA signals. Figure 4(d) presents the variation of measured PA signals (obtained through averaging PA-signals over target regions) with mechanical properties (Young's modulus). From Figs. 4(a)-4(d), it is observed that (short pulse) light-stimulated PA signals increase with an 



Fig. 5 (a) Three-dimensionally reconstructed image of PA signal for raster scanning in sample (2). (b) Line plot showing variation of detected PA signals along a midline in the first frame of 3-D reconstructed image [marked line as shown in (a)]. (c) Dependence of PA signals on size of targets embedded in background.

increase in elastic property (say, Young's modulus), which is not linear.

Figure 5(a) gives a 3-D reconstructed image of PA signals while the variation of PA signals along a line [as depicted in Fig. 5(a)] in the first frame is shown in Fig. 5(b). The figure demonstrates that the strength of measured PA-signals is similar for all targets [ $T_{1}$ to $T_{4}$ shown in Fig. 3(b)]. Note that targets $\left(T_{1}\right.$ to $T_{4}$ ) in the second sample [Fig. 3(b)] had no contrast in elastic and optical properties (among themselves as well as the background) while differing only in their sizes or dimensions of the cross-section in $x z$ plane. Variation of measured PA signals with size of target cross-section is also shown in Fig. 5(c), and this result demonstrates that measured PA signals are not dependent on the size of the target of interest.

Figure 6 shows the results of experiments being conducted in the first sample [shown in Fig. 3(a)] for studying variation in PA-signal strength with a change in elastic coefficient $(E)$ of targets. ${ }^{85,89}$ Two sets of experiments were conducted, in which two different focusing US transducers with operating frequencies of $50 \mathrm{MHz}$ [experimental results are shown in Figs. 6(a)6(c)] and 3.5 MHz [shown in Figs. 6(d)-6(f)] were used alternately as an acoustic sensor. Figure 6(a) presents a reconstructed 2-D image that is representative of PA-signal strength obtained through raster scanning of the second sample [Fig. 3 (b)] in the middle portion along the $x$-direction and the corresponding 3-D image is shown in Fig. 6(a). From images depicting the strength of detected PA signals (corresponding to $T_{2}$ through $T_{6}$ ), it is observed that targets are clearly detectable (though feebly for $T_{6}$ ) in the background while $T_{1}$ is not detectable in the background without any contrast in measured PA signals. Figure 6(c) displays line-plots of variation in the strength of PA signals along a marked line [indicated in Fig. 6(b)]. In consideration to the description made above [for first sample, 3(a)], observed contrast in the measured PA signal strength for targets $\left(T_{2}\right.$ to $\left.T_{6}\right)$-having contrast in Young's modulus $(E)$ relative to background elastic coefficient while maintaining no contrast in optical properties-is due to contrast in elastic property of targets relative to that of background. On the other hand, contrast observed for $T_{2}$-with no contrast in $E$ while tailoring contrast in $\mu_{a}$-is provided due to contrast in $\mu_{a}$. From the figure, target $T_{1}$ gives no contrast in measured PA signals as there is no contrast in optical as well



Fig. 6 For experiments performed in second sample, (a) and (d) 3-D and (b) and (e) 2-D images representative of strength of PA signals as detected by focusing US transducers [operating frequencies $50 \mathrm{MHz}$ (a)-(c) and 3.5 MHz (d)-(f)]. Line-plots corresponding to the marked lines are shown in $(\mathrm{c})$ and $(\mathrm{f})$.

as elastic properties. From experimental results, it is observed that the strength of measured PA signals increases with an increase in contrast level of elastic coefficient of embedded targets (that are coupled to background). The underlying reason is due to change in natural frequency of vibration of elastically coupled system, constituted by background and targets, toward operating frequency of transducer in higher range $(\sim 50 \mathrm{MHz}){ }^{89}$ Figures 6(d) and 6(e) present 3-D and 2-D images obtained from scanning of the second sample with ultrasound transducer of operating frequency of $3.5 \mathrm{MHz}$, whereas Fig. 6(f) gives variation in measured strength of PA signals along a line marked passing through targets (as indicated). From the figures, it is evident that measured strength of PA signals, as detected by $3.5 \mathrm{MHz}$, gives contrast only for a target $T_{2}$ while PA signal strengths corresponding to targets $T_{1}$ to $T_{6}$ (with exception of $T_{2}$ ) have no contrast irrespective of contrast level in target elastic coefficients. This is due to the increase in natural frequency of mechanical vibration of an elastically coupled system that is far away from the transducer operating frequency ( $3.5 \mathrm{MHz})$.

Figure 7(b) gives - as shown in our reported study ${ }^{85}$ for experiments being performed in the third sample [Fig. 3(c)]a variation in measured PA-signal strength with transducer

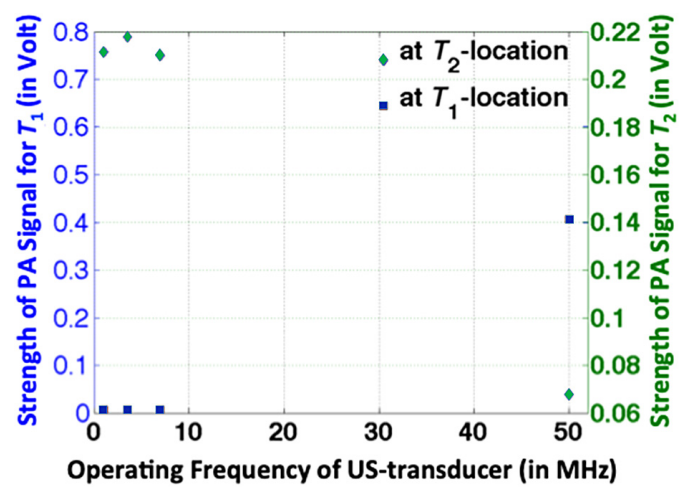

Fig. 7 Variation of PA signals as obtained from target regions of third sample [Fig. 3(c)] with operating frequency of (focusing) US transducer. 
operating frequency. For $t_{1}$ that has contrast in $E$, measured signal strength is relatively high for higher operating frequency $(\sim 50 \mathrm{MHz})$ in comparison to that of lower range $(\sim 3.5 \mathrm{MHz})$. PA-signal strength is weak (undetectable) in the background. For $t_{2}$ having $\mu_{a}$-contrast, PA-signal strength is higher for operating frequency in the lower range $(\sim 3.5 \mathrm{MHz})$ than that of ultrasound sensor with higher operating frequency $(\sim 50 \mathrm{MHz})$. From the figure, we observe a shift in the frequency response of measured PA-signal strength with variation in nature of contrast-either elastic property $(E)$ or optical absorption coefficient $\left(\mu_{a}\right)$-in targets. Experimental results imply that US transducer of higher operating frequency $(\sim 50 \mathrm{MHz})$ is demanded to detect PA signals from targets having contrast in $E$, instead of conventionally used US transducer of operating frequency in the lower range $(\sim 1 \mathrm{MHz})$ for detecting PA signals from targets having contrast in $\mu_{a}$.

\subsubsection{Photoacoustic tomography elastography imaging}

Pengfei et al. ${ }^{62,91,92}$ developed a PAT imaging system — that they called quantitative photoacoustic elastography (QPAE) imaging system-for recovery of tissue elastic property noninvasively and nondestructively. Validation studies were carried out both in tissue-mimicking phantom (gelatin) and animal model (mouse). The study demonstrated the feasibility of PAE — with an accuracy less than $5.2 \%$ in comparison to theoretical values-by imaging strains induced in various layers of phantoms with various stiffness values. Presented experimental results provide a mapping of fat and muscle based on measured elastic property contrast with the experiment being conducted (in-vivo) on a mouse leg and results were compared with that of USE imaging modality being performed simultaneously.

QPAE imaging system - as shown in Fig. 8 (the schematic diagram is reproduced with permission from Refs. 91 and 92)employed a commercially available linear-array photoacoustic computed tomography system. In this study, for PA excitation, a pulse laser beam (pulse width $\sim 10 \mathrm{~ns}$ and optical wavelength $\sim 680 \mathrm{~nm}$ ) at a pulse repetition rate of $20 \mathrm{~Hz}$ is generated from the pulse laser source. For detection of pulse laserinduced PA-signals, a linear ultrasonic transducer array (LZ250, VisualSonics Inc.) of 256 elements with center frequency $\sim 21 \mathrm{MHz}$ was employed. As shown in Fig. 8, a fiber bundle-that was adopted for coupling light from the laser source to tissue sample-is split into two rectangular bars and these two bars are mounted on each side of the linear transducer array. The imaging system provides spatial resolutions $\sim 119 \mu \mathrm{m}$ (in lateral direction), $\sim 86 \mu \mathrm{m}$ (in the axial direction), and $\sim 1.2 \mathrm{~mm}$ (in elevational direction) with a frame rate of acquisition of 2-D image [5 $\mathrm{Hz}$ as only one quarter (i.e., 64) of 256-transducer elements are utilized in one laser pulse excitation and a full set of data from all of 256 elements is used for generating a 2-D image]. An aluminum plate-larger than sample-was used to exert an axial compressive force on tissue sample of interest and compression plate was equipped with an imaging window at center so as to allow laser-induced PA signals to be detected by transducer array. A manually controlled translation stage was adopted to provide compression to imaging object resting against a rigid object holder. Reading from a high precision digital weighing scale (S200, Ohaus), on which object and object holder was kept, was used to calculate compression stress. The total displacement of tissue sample was obtained from measurement in manual stage readings while stress acting on the sample is estimated (from readings before and after compression) as follows:

$\sigma=\frac{g\left(m_{a}-m_{b}\right)}{a}$

where $\sigma$ is compression stress, $g$ is acceleration due to gravity, $m_{a}$ and $m_{b}$ are scale readings before and after compression, and $a$ is the cross-sectional area. The above-mentioned QPAE imaging system is similar to compressional elastography, which is among other variants of US- and OCT-based elastography imaging modalities that include quasistatic, dynamical nonresonant, dynamical resonant, nearly homogeneous (applied) stress, and strongly localized excitation. Only the specific difference is that, instead of detecting and characterizing scattered ultrasound (in USE) or optical (in OCE) waves, which are adopted as tissue interrogating signals, optically irradiated absorbing particles generate acoustic signals and these light-induced acoustic waves are adopted as interrogating signals.

Experiments were conducted in tissue-mimicking gelatinbased phantoms with various concentrations of 40 to $100 \mathrm{~g} / \mathrm{L}$ (at the step of $20 \mathrm{~g} / \mathrm{L}$ ) that provide mechanical properties of the

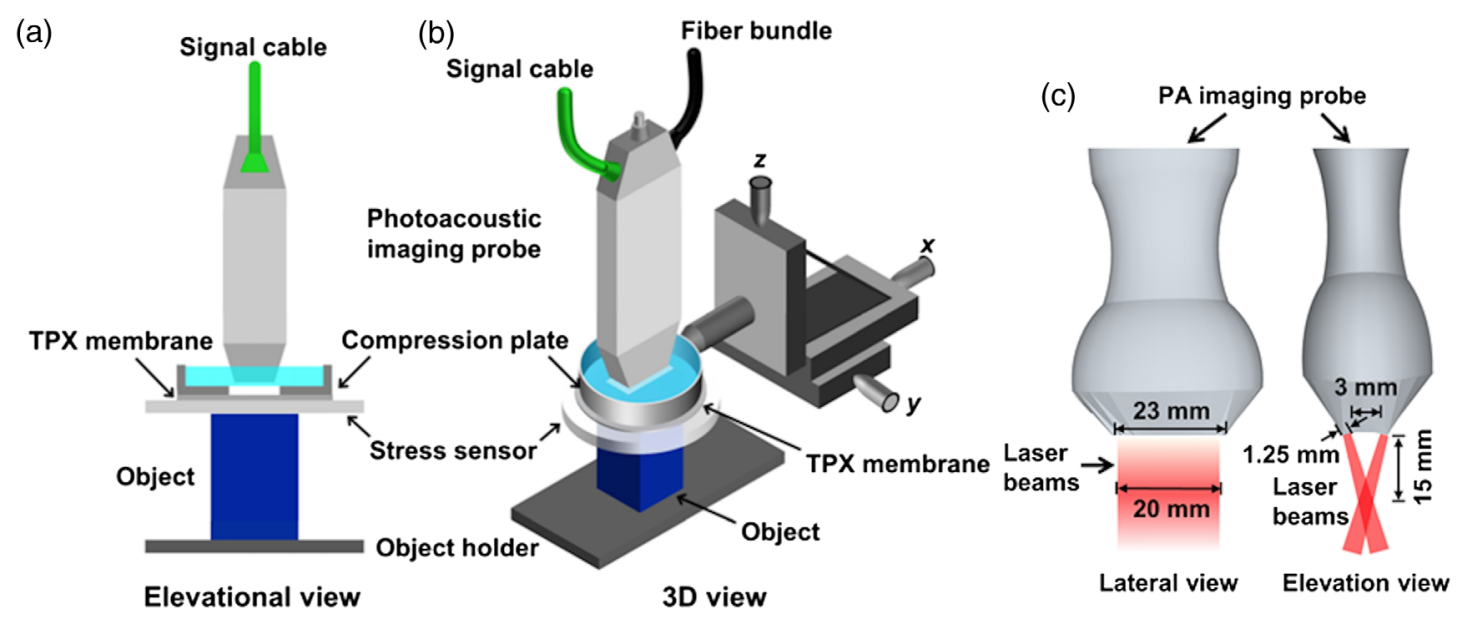

Fig. 8 Schematic diagram of QPAE imaging system: (a) at an elevational view and (b) at 3-D view. Lateral and elevational view of illumination of pulse laser beam in QPAE imaging system [(a) and (b)] are depicted in (c). (Figures are reproduced with permission.) 
sample at different values. To provide optical contrast for PA imaging, microspheres $(\sim 50 \mu \mathrm{m})$ - at a concentration of $\sim 5 / \mathrm{mm}^{3}$ - are mixed in gelatin phantom. Microsphereembedded gelatin phantoms were imaged individually before and after mechanical compression with external stress (of $53 \mathrm{~Pa}$ ) using manually controlled mechanism mentioned above. Timeresolved (A-line) signals - obtained corresponding to each of the transducer elements-before [as shown in Fig. 9(a)] and after compression [presented in Fig. 9(a)] were cross-correlated to estimate axial displacement due to compression. A cross-sectional map of displacements of microspheres after compression is presented in Fig. 9(c). In this cross-correlation-based method, cross-correlation (over a short length of $90 \mu \mathrm{m}$ ) of A-linescorresponding to before and after compression-was computed and displacements obtained from microspheres at each depth were then averaged. The slope of linear fitting - as shown in Fig. 9(d)—of measured variation of displacement against depth, i.e., the magnitude of the average gradient of displacement, gives average strain of each gelatin phantom. Five sets of experiments are repeated for each of the four gelatin phantoms and measurement of strain with respect to gelatin concentration is plotted in Fig. 9(e).

Experimental studies were extended to conduct experiments in (in vivo) animal model-in mouse leg in conformity with laboratory animal protocols approved by the Animal Studies Committee at Washington University in St. Louis-for validation of elastography imaging system to preclinical studies and its applications. In the experiments, the mouse leg was imaged before [shown in Fig. 10(a)] and after [shown in Fig. 10(b)] applying an external compression force $(\sim 12 \mathrm{mN})$. From these two images, a displacement image was obtained using the above-mentioned cross-correlation technique, and it is shown in Fig. 10(c). Raw strain image was then superimposed on structural PA image [Fig. 10(e)]. Regions of softer tissue, i.e., fat, give larger strains, which were validated by USE using the same linear-array imaging probe, which showed a similar distribution of strains, as depicted in Figs. 10(d) and 10(f).

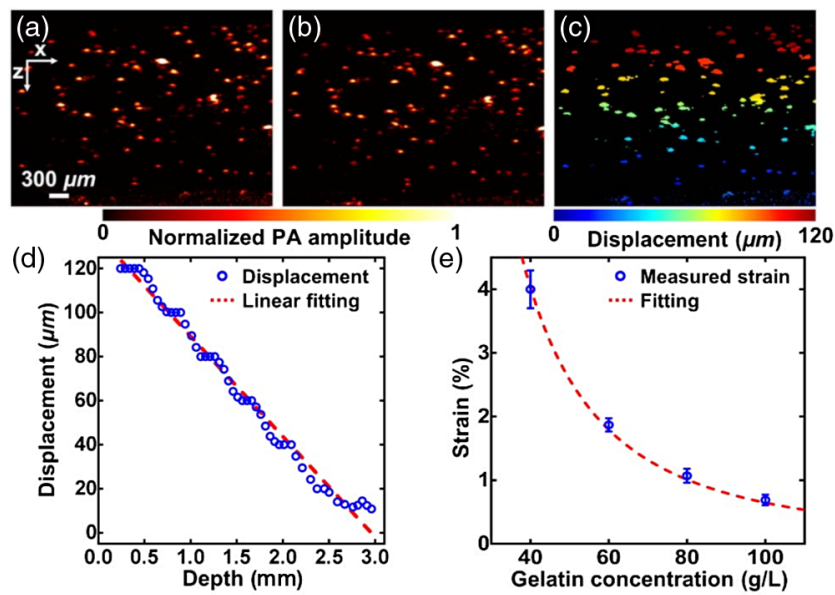

Fig. 9 Measurement of strain being carried out in gelatin phantom by PAE: (a) and (b) PA images of a bilayer gelatin phantom mixed with $50 \mu \mathrm{m}$ microspheres acquired (a) before and (b) after compression. (c) Mapping of displacement of embedded microspheres obtained from Figs. 9(a) and 9(b). (d) Variation of average displacement versus depth. Data were fitted by a linear function for each layer. (e) Measured strains of gelatin phantoms with $4 \%, 6 \%, 8 \%$, and $10 \%$ concentration in weight. Figure shows a fit of the curve with quadratic model. (Figures are reproduced with permission.)
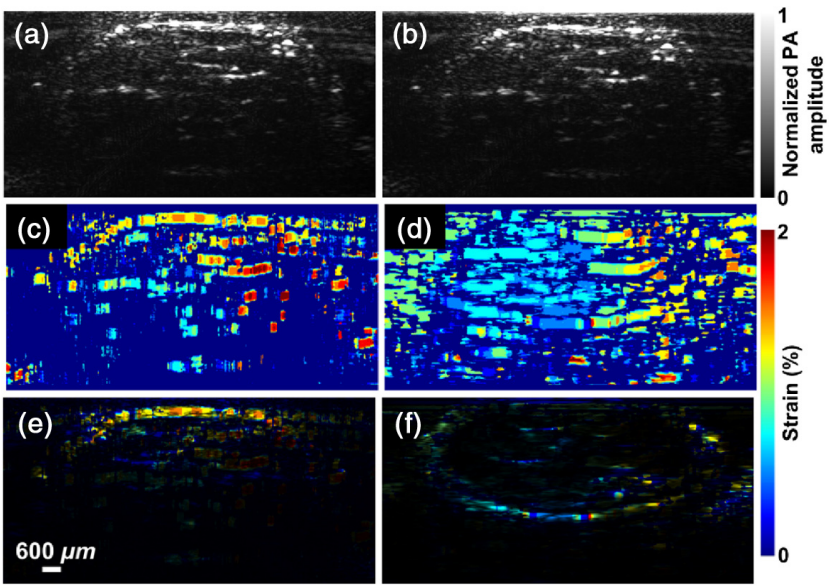

Fig. 10 PAE images of a mouse leg in vivo (a) before and (b) after compression. (c) Strain image of mouse leg (in vivo). (d) Strain image of mouse leg obtained by USE. (e) Strain image of mouse leg obtained by PAE superimposed on structural PA image. (f) Strain image of mouse leg obtained by USE on structural ultrasound image. (Figures are reproduced with permission.)

\subsubsection{Challenges for photoacoustic elastography imaging and some technological approaches}

PAEI is associated with certain challenges that may be drawn as follows: (1) low PA signal contrast - as detected by acoustic (ultrasound transducer) sensor kept on sample boundarydue to variation of mechanical elastic property distribution of tissue in comparison to that of optical absorption coefficient, ${ }^{85,89}$ (2) quantitative assessment, i.e., to quantify measurement, of elastic property; and (3) discrimination of contrast in PA signal due to elastic property variation from that of other parameters (including optical absorption coefficient).

External mechanical stimulation of tissues combined with correlation-based technique - as done in USE imaging ${ }^{6}$ - is a potential technological approach to enhance signal contrast. Acoustic burst (mechanical) stimulation technique, ${ }^{52}$ alternative to continuous external excitation, is shown to improve modulated signals significantly due to increased mechanical modulation of tissue-over an ultrasound insonified region-with higher acoustic radiation force driven by an external (tightly) focusing ultrasound transducer. As done in Ref. 60, Young's modulus can be estimated from resonance peak-corresponding to natural frequency of vibration of tissue materials over ultrasound insonified region-observed in measurement and plots of PA signal strength against the operating frequency of external (ultrasound) mechanical stimulation. In the end, in comparison to the similarity in nature of externally induced mechanical stimulation and investigation of interrogating signals (say, light and acoustic), existing technologies for quantitative recovery of elastic properties (more specifically, in USE and OCE) may be adapted to PAE.

\section{Conclusion}

We have presented a review of studies on imaging of elastic properties of soft tissues, as reported in the literature to date. It was found that reported studies of the imaging modalityboth microscopy and tomography - are limited and the research study in this area is in the nurture stage with the first study being reported only recently (in 2011). It is a potential research area with significant implications both in basic sciences and clinical 
applications. The article draws out challenges pertaining to PAEI as well as technological approaches to address the challenges, which may present potential research problems for researchers working in biomedical domains. Last, in comparison with USE and OCT elastography, PAE is yet in the very beginning of its development, and there are promising scopes for its rapid progress.

\section{Disclosures}

The authors have no relevant financial interests in this article and no other potential conflicts of interest to disclose. The experiments that were conducted in in-vivo animal model-more specifically, in mouse leg-were in conformity with laboratory animal protocols approved by the Animal Studies Committee at Washington University in St. Louis.

\section{References}

1. J. F. Greenleaf, M. Fatemi, and M. Insana, "Imaging of elastic properties of tissue-a review," Ultrason. Med. Biol. 22(8), 959-977 (1996).

2. A. Samani, J. Zubovits, and D. Plewes, "Elastic moduli of normal and pathological human breast tissues: an inversion-technique-based investigation of 169 samples," Phys. Med. Biol. 52, 1565-1576 (2007).

3. Y. C. Fung, Biomechanics: Mechanical Properties of Living Tissue, Springer-Verlag, New York (1993).

4. T. Krouskop et al., "Elastic moduli of breast and prostate tissues under compression," Ultrason. Imaging 20, 260-274 (1998).

5. B. Shraiman, "Mechanical feedback as a possible regulator of tissue growth," Proc. Natl. Acad. Sci. U.S.A. 102, 3318-3323 (2005).

6. J. Ophir et al., "Elastography: a quantitative method for imaging the elasticity of biological tissues," Ultrason. Imaging 13, 111-134 (1991).

7. J. C. Eric et al., "Young's modulus measurements of soft tissues with application to elasticity imaging," Ultrason. Imaging 43(1), 191-194 (1996).

8. J. F. Greenleaf, M. Fatemi, and M. Insana, "Selected methods for imaging elastic properties of biological tissues," Annu. Rev. Biomed. Eng. 5(1), 57-78 (2003).

9. A. P. Sarvazyan et al., "Biophysical bases of elasticity imaging," Acoust. Imaging 21, 223-240 (1995).

10. R. Muthupillai et al., "Magnetic resonance elastography by direct visualization of acoustic strain waves," Science 269, 1854-1857 (1995).

11. M. Fatemi, A. Manduca, and J. F. Greenleaf, "Imaging elastic properties of biological tissues by low-frequency harmonic vibration," Proc. IEEE 91(10), 1503-1519 (2003).

12. M. L. Palmeri, K. D. Frinkley, and K. R. Ninghtingale, "Experimental studies of the thermal effects associated with radiation force imaging of soft tissue," Ultrason. Imaging 26, 100-114 (2004).

13. E. J. Chen et al., "Young's modulus measurements of soft tissues with application to elasticity imaging," IEEE Trans. Ultrason. Ferroelectr. Freq. Control 43(1), 191-194 (1996).

14. A. L. McKnight et al., "MR elastography of breast cancer: preliminary results," Am. J. Roentgenol. 178, 1411-1417 (2002).

15. J. Ophir et al., "Elastography: imaging the elastic properties of soft tissues with ultrasound," J. Med. Ultrasonics 29, 155-171 (2002).

16. I. Cespedes et al., "Elastography—elasticity imaging using ultrasound with application to muscle and breast in-vivo," Ultrason. Imaging $\mathbf{1 5}$, 73-88 (1993).

17. J. Bercoff et al., "In vivo breast tumor detection using transient elastography," Ultrasound Med. Biol. 29, 1387-1396 (2003).

18. K. M. Yogesh, J. G. Kevin, and L. E. Richard, "Magnetic resonance elastography: a review," Clin. Anat. 23(5), 497-511 (2010).

19. K. V. Sudhakar, Y. Meng, and L. E. Richard, "Magnetic resonance elastography: magnetic resonance elastography of liver: technique, analysis and clinical applications," J. Magn. Reson. Imaging 37(3), 544-555 (2013).
20. E. W. Van Houten et al., "Initial in vivo experience with steady-state subzone-based MR elastography of the human breast," J. Magn. Reson. Imaging 17, 72-85 (2003).

21. A. Sinkus et al., "Viscoelastic shear properties of in vivo breast lesions measured by MR elastography," Magn. Reson. Imaging 23, 159-165 (2005).

22. J. M. Schmitt, "OCT elastography: imaging microscopic deformation and strain of tissue," Opt. Express 3(6), 199-211 (1998).

23. A. Nahas et al., "3D static elastography at the micrometer scale using full field OCT," Biomed. Opt. Express 4(6), 2138-2148 (2013).

24. V. Y. Zaitsev et al., "Deformation-induced speckle-pattern evolution and feasibility of correlational speckle tracking in optical coherence elastography," J. Biomed. Opt. 20(7), 075006 (2015).

25. B. F. Kennedy et al., "Strain estimation in phase-sensitive optical coherence elastography," Biomed. Opt. Express 3(8), 1865-1879 (2012).

26. A. L. Matveyev et al., "Vector method for strain estimation in phasesensitive optical coherence elastography," Laser Phys. Lett. 15(8), 065603 (2018).

27. K. M. Kennedy et al., "Quantitative micro-elastography: imaging of tissue elasticity using compression optical coherence elastography," Sci. Rep. 5, 15538 (2015).

28. V. Y. Zaitsev et al., "Practical obstacles and their mitigation strategies in compressional optical coherence elastography of biological tissues," J. Innovative Opt. Health Sci. 10(6), 1742006 (2017).

29. W. M. Allen et al., "Wide-field optical coherence micro-elastography for intraoperative assessment of human breast cancer margins," Biomed. Opt. Express 7(10), 4139-4153 (2017).

30. L. Chin et al., "Simplifying the assessment of human breast cancer by mapping a micro-scale heterogeneity index in optical coherence elastography," J. Biophotonics 10, 690-700 (2017).

31. A. A. Plekhanov et al., "Optical coherence elastography for non-invasive monitoring of tumor elasticity under chemotherapy: pilot study," Sovrem. Tehnol. 10(3), 43-49 (2018).

32. J. Fu et al., "Depth-resolved full-field measurement of corneal deformation by optical coherence tomography and digital volume correlation," Exp. Mech. 56, 1203-1217 (2016).

33. V. Y. Zaitsev et al., "Optical coherence elastography for strain dynamics measurements in laser correction of cornea shape," J. Biophotonics 10(11), 1450-1463 (2017).

34. V. Y. Zaitsev et al., "Revealing structural modifications in thermomechanical reshaping of collagenous tissues using optical coherence elastography," J. Biophotonics 10(11), 1450-1463 (2017).

35. Y. Qiu et al., "Nonlinear characterization of elasticity using quantitative optical coherence elastography," Biomed. Opt. Express 7(11), 4702-4710 (2016).

36. A. A. Sovetsky et al., "Manually-operated compressional optical coherence elastography with effective aperiodic averaging: demonstrations for corneal and cartilaginous tissues," Laser Phys. Lett. 15, 085602 (2018).

37. B. F. Kennedy, P Wijesinghe, and D. D. Sampson, "The emergence of optical elastography in biomedicine," Nat. Photonics 11(4), 215-221 (2017).

38. A. K. Mitchell et al., "Optical coherence elastography in ophthalmology," J. Biomed. Opt. 22(12), 121720 (2017).

39. K. Larin and D. D. Sampson, "Optical coherence elastography-OCT at work in tissue biomechanics," Biomed. Opt. Express 8(2), 11721202 (2017).

40. X. Liang et al., "Optical micro-scale mapping of dynamic biomechanical tissue properties," Opt. Express 16(15), 11052-11065 (2008).

41. T. S. Ralston et al., "Interferometric synthetic aperture microscopy," Nat. Phys. 3, 129-134 (2007).

42. T. S. Ralston et al., "Inverse scattering for high-resolution interferometric microscopy," Opt. Lett. 31, 3585-3587 (2006).

43. J. G. Fujimoto, "Optical coherence tomography for ultrahigh resolution in vivo imaging," Nat. Biotechnol. 21, 1361-1367 (2003).

44. D. Huang et al., "Optical coherence tomography," Science 254, 1178-1181 (1991).

45. G. J. Tearney et al., "In vivo endoscopic optical biopsy with optical coherence tomography," Science 276, 2037-2039 (1997).

46. M. Wojtkowski et al., "Ultrahigh-resolution, high-speed, Fourier domain optical coherence tomography and methods for dispersion compensation," Opt. Express 12, 2404-2422 (2004). 
47. J. F. Andrew, L. F. Jeffrey, and S. G. Brian, "Lung strain profiles using computed tomography elastography," in 26th Annu. Int. Conf. IEEE Eng. Med. and Biol. Soc., pp. 1545-1548 (2004).

48. G. Tanya and A. Haim, "A method for characterization of tissue elastic properties combining ultrasonic computed tomography with elastography," J. Ultrasound Med. 29, 387-398 (2010).

49. S. Takehisa, H. Mineyuki, and O. Sadao, "CT elastography: a pilot study via a new endoscopic tactile sensor," Open J. Biophys. 4, 22-28 (2014).

50. D. B. Mehmet et al., "Comparison of computed tomography densitometry and shear wave elastography velocity measurements for evaluation of the liver volume in the nonalcoholic fatty liver disease," Int. J. Clin. Exp. Med. 9(6), 10159-10169 (2016).

51. M. S. Singh, K. Rajan, and R. M. Vasu, "Estimation of elasticity map of soft biological tissue mimicking phantom using laser speckle contrast analysis," J. Appl. Phys. 109, 104704 (2011).

52. M. S. Singh, K. Rajan, and R. M. Vasu, "Intense acoustic burst ultrasound modulated optical tomography for elasticity mapping of soft biological tissue mimicking phantom: a laser speckle contrast analysis study," Proc. SPIE 9040, 90401S (2014).

53. P.-Y. Chao et al., "Shear wave elasticity measurements of threedimensional cancer cell cultures using laser speckle contrast imaging," Sci. Rep. 8(1), 1-10 (2018).

54. P.-Y. Chao et al., "Laser speckle contrast shear wave imaging of threedimensional cancer metastasis model," in IEEE Int. Ultrason. Symp. (2017).

55. Y.-S. Yang, P.-Y. Chao, and P.-C. Li, "Tomographic shear wave imaging: feasibility study," in IEEE Int. Ultrason. Symp. (2017).

56. P.-Y. Chao and P.-C. Li, "Three-dimensional shear wave imaging based on full-field laser speckle contrast imaging with onedimensional mechanical scanning," Opt. Express 24(17), 18860-18871 (2016).

57. C.-T. Li et al., "3D cell mechanobiology study using shear wave elasticity imaging," in IEEE Int. Ultras. Symp. (2014).

58. P.-L. Kuo et al., "Shear-wave elasticity measurements of threedimensional cell cultures for mechanobiology," J. Cell Sci. 130, 292-302 (2016).

59. M. S. Singh et al., "Assessment of ultrasound modulation of near infrared light on the quantification of scattering coefficient," Med. Phys. 37(7), 3744-3751 (2010).

60. M. S. Singh et al., "Quantitative estimation of mechanical and optical properties from ultrasound assisted optical tomography data," J. Biomed. Opt. 17(10), 101507 (2012).

61. M. S. Singh et al., "Ultrasound modulation of coherent light in a multiple-scattering medium: experimental verification of nonzero average phase carried by light," Biomed. Opt. Express 3(9), 2100-2110 (2012).

62. H. Pengfei et al., "Photoacoustic elastography," Opt. Lett. 41(4), 725-728 (2016).

63. K. J. Parker, M. M. Doyley, and D. J. Rubens, "Imaging the elastic properties of tissue: the 20 year perspective," Phys. Med. Biol. 57, 5359-5360 (2012).

64. B. S. Garra et al., "Elastography of breast lesions: initial clinical results," Radiology 202, 79-86 (1997).

65. A. Samani, J. Bishop, and D. B. Plewes, "A constrained modulus reconstruction technique for breast cancer assessment," IEEE Trans. Med. Imaging 20, 877-885 (2001).

66. J. T. Bushberg et al., Essential Physics of Medical Imaging, 3rd ed., North American Edition, Lippincott Williams \& Wilkins, Philadelphia, Pennsylvania (2012).

67. L. S. Wilson, D. E. Robinson, and M. J. Dadd, "Elastography-the movement begins," Phys. Med. Biol. 45, 1409-1421 (2000).

68. R. Sinkus et al., "Imaging anisotropic and viscous properties of breast tissue by magnetic resonance-elastography," Magn. Reson. Med. 53, 372-387 (2005).

69. R. Huber, D. C. Adler, and J. G. Fujimoto, "Buffered Fourier domain mode locking: unidirectional swept laser sources for optical coherence tomography imaging at 370,000 lines/s," Opt. Lett. 31, 2975-2977 (2006).

70. E. M. Strohm, M. J. Moore, and M. C. Kolios, "Single cell photoacoustic microscopy: a review," IEEE J. Sel. Top. Quantum Electron. 22(3), 137-151 (2016).

71. L. V. Wang and S. Hu, "Photoacoustic tomography: in vivo imaging from organelles to organs," Science 335, 1458-1462 (2012).
72. https://www.pubfacts.com/search/photoacoustic+imaging.

73. P. K. Upputuri and P. Manojit, "Recent advances toward preclinical and clinical translation of photoacoustic tomography: a review," J. Biomed. Opt. 22(4), 041006 (2017).

74. S. Jaejung et al., "'Smart' gold nanoparticles for photoacoustic imaging: an imaging contrast agent responsive to the cancer microenvironment and signal amplification via $\mathrm{pH}$-induced aggregation," Chem. Commun. 52, 8287-8290 (2016).

75. E. E. Connor et al., "Gold nanoparticles are taken up by human cells but do not cause acute cytotoxicity," Small 1, 325-327 (2005).

76. H. Jiang, Z. Yuan, and X. Gu, "Spatially varying optical and acoustic property reconstruction using finite-element-based photoacoustic tomography," J. Opt. Soc. Am. 23, 878-888 (2006).

77. J. Shah et al., "Photoacoustic imaging and temperature measurement for photothermal cancer therapy," J. Biomed. Opt. 13, 034024 (2008).

78. S. Shriram et al., "Intravascular photoacoustic imaging using an IVUS imaging catheter," IEEE Trans. Ultrason. Ferroelectr. Freq. Control 54(5), 978-986 (2007).

79. W. Xia et al., "Handheld real-time LED-based photoacoustic and ultrasound imaging system for accurate visualization of clinical metal needles and superficial vasculature to guide minimally invasive procedures," Sensors 18, 1394 (2018).

80. A. Buehler et al., "Real-time handheld multispectral optoacoustic imaging," Opt. Lett. 38(9), 1404-1406 (2013).

81. Z. Zhang et al., "Subdiffraction-limited second harmonic photoacoustic microscopy based on nonlinear thermal diffusion," Opt. Lett. 43(10), 2336-2339 (2018).

82. L. Xi, L. Zhou, and H. Jiang, "C-scan photoacoustic microscopy for invivo imaging of Drosophila pupae," Appl. Phys. Lett. 101, 013702 (2012).

83. M. S. Singh and H. Jiang, "Estimating both direction and magnitude of flow velocity using photoacoustic microscopy," Appl. Phys. Lett. 104, 253701 (2014).

84. G. Gao, S. Yang, and D. Xing, "Viscoelasticity imaging of biological tissues with phase-resolved photoacoustic measurement," Opt. Lett. 36(17), 3341-3343 (2011).

85. M. S. Singh and H. Jiang, "Elastic property attributes to photoacoustic signals: an experimental phantom study," Opt. Lett. 39(13), 3970-3973 (2014).

86. N. Wadamori, "Non-restrained measurement of Young's modulus for soft tissue using a photoacoustic technique," Appl. Phys. Lett. 105, 103707 (2014).

87. Y. Zhao et al., "Simultaneous optical absorption and viscoelasticity imaging based on photoacoustic lock-in measurement," Opt. Lett. 39(9), 2565-2568 (2014).

88. Y. L. Pengfei and Z. Yuan, "Multi-spectral photoacoustic elasticity tomography," Biomed. Opt. Express 7(9), 3323-3334 (2016).

89. M. S. Singh and H. Jiang, "Ultrasound (US) transducer of higher operating frequency detects photoacoustic (PA) signals due to the contrast in elastic property," AIP Adv. 6, 025210 (2016).

90. Q. Wang et al., "Quantitative photoacoustic elasticity and viscosity imaging for cirrhosis detection," Appl. Phys. Lett. 112, 211902 (2018).

91. H. Pengfei et al., "Quantitative photoacoustic elastography of Young's modulus in humans," Proc. SPIE 10064, 100640B (2017).

92. H. Pengfei et al., "Photoacoustic tomography of vascular compliance in humans," J. Biomed. Opt. 20(12), 126008 (2015).

93. M. Scholz et al., "Vibrography during tumor neurosurgery," J. Ultrasound Med. 24, 985-992 (2005).

94. Z. Xu, R. Paparcone, and M. J. Buehler, "Alzheimer's Ab(1-40) amyloid fibrils feature size-dependent mechanical properties," Biophys. $J$. 98, 2053-2062 (2010).

95. P. J. van den Berg, K. Daoudi, and W. Steenbergen, "Review of photoacoustic flow imaging: its current state and its promises," Photoacoustics 3, 89-99 (2015).

96. X. Jun and L. V. Wang, "Small-animal whole-body photoacoustic tomography: a review," IEEE Trans. Biomed. Imaging 61(5), 13801389 (2014).

97. S. Manohar and R. Daniel, "Photoacoustics: a historical review," Adv. Opt. Photonics 8(4), 586-617 (2016).

98. M. Li, Y. Tang, and J. Yao, "Photoacoustic tomography of blood oxygenation: a mini review," Photoacoustics 10, 65-73 (2018). 
99. P. Beard, "Biomedical photoacoustic imaging," Interface Focus 1, 602-631 (2011).

100. N. Vasilis, "Going deeper than microscopy: the optical imaging Frontier in biology," Nat. Methods 7(8), 603-614 (2010).

101. S. Mallidi, G. P. Luke, and S. Emelianov, "Photoacoustic imaging in cancer detection, diagnosis, and treatment guidance," Trends Biotechnol. 29(5), 213-221 (2011).

102. G. R. Steffen, A. C. Boccara, and W. Steenbergen, "State-of-the-art of acousto-optic sensing and imaging of turbid media," J. Biomed. Opt. 17(4), 040901 (2012).

103. B. Joanna et al., "Photoacoustic imaging using genetically encoded reporters: a review," J. Biomed. Opt. 22(7), 070901 (2017).

104. L. V. Wang and L. Gao, "Photoacoustic microscopy and computed tomography: from bench to bedside," Аnпи. Rev. Biomed. Eng. 16, $155-185$ (2014).

105. X. Cai et al., "Photoacoustic microscopy in tissue engineering," Mater. Today 16(3), 67-77 (2013).

106. J. Yao and L. V. Wang, "Sensitivity of photoacoustic microscopy," Photocoustics 2(2), 87-101 (2014).

107. B. Hamilton and A. Clain, Hamilton Bailey's Demonstrations of Physical Signs in Clinical Surgery, pp. 16-17, John Wright and Sons, Bristol (1986).

108. R. M. Lerner, S. R. Huang, and K. J. Parker, “'Sonoelasticity' images derived from ultrasound signals in mechanically vibrated tissues," Ultrasound Med. Biol. 16, 231-239 (1990).

109. K. J. Parker et al., "Tissue response to mechanical vibrations for sonoelasticity imaging," Ultrasound Med. Biol. 16, 241-246 (1990).

110. T. A. Krouskop, D. R. Dougherty, and S. F. Levinson, "A pulsed Doppler ultrasonic system for making noninvasive measurements of the mechanical properties of soft tissue," J. Rehabil. Res. Dev. 24, 1-8 (1987).

111. S. F. Levinson, "Ultrasound propagation in anisotropic soft tissues: the application of linear elastic theory," J. Biomech. 20, 251-260 (1987).

112. L. N. Bohs and G. E. Trahey, "An efficient technique for two-dimensional ultrasonic velocity imaging," Ultrason. Imaging 11, 133 (1989).

113. S. Yagi and K. Nakayama, "Local displacement analysis of inhomogeneous soft tissue by spatial correlation of RF echo signals," in Proc. World Fed. Ultrasound Med. and Biol., Washington, DC, p. 133 (1988).

114. R. J. Dickinson and C. R. Hill, "Measurement of soft tissue motion using correlation between A-scans," Ultrasound Med. Biol. 8, 263-271 (1982).

115. P. G. M. De Jong et al., "Determination of tissue motion velocity by correlation interpolation of pulsed ultrasonic echo signals," Ultrason. Imaging 12, 84-98 (1990).

116. G. E. Trahey, S. M. Hubbard, and O. T. Von Ramm, "Angle independent blood flow detection by frame-to-frame correlation of B-mode images," Ultrasonics 26, 271-276 (1988).

117. L. S. Wilson and D. E. Robinson, "Ultrasonic measurement of small displacements and deformations of tissues," Ultrason. Imaging 4, 71-82 (1982).

118. M. Tristam et al., "Ultrasonic study of in vivo kinetic characteristics of human tissues," Ultrasound Med. Biol. 12, 927-937 (1986).

119. R. S. Adler et al., "Quantitative tissue motion analysis of digitized M-mode images: gestational differences of fetal lung," Ultrasound Med. Biol. 16(6), 561-569 (1990).

120. W. K. Chu and D. E. Raeside, "Fourier analysis of the echo-cardiogram," Phys. Med. Biol. 23, 100-105 (1978).

121. M. Tristam et al., "Application of Fourier analysis to clinical study of patterns of tissue movement," Ultrasound Med. Biol. 14(8), 695-707 (1988).

122. Y. Yamakoshi, T. Sato, and T. Sato, "Ultrasonic imaging of internal vibration of soft tissue under forced vibration," J. Acoust. Soc. Am. 84, S139 (1988).

123. Y. Yamakoshi, T. Sato, and T. Sato, "Ultrasonic imaging of internal vibration of soft tissue under forced vibration," IEEE Trans. Ultrason. Ferroelectr. Freq. Control 37(2), 45-53 (1990).

124. J. L. Gennisson et al., "Ultrasound elastography: principles and techniques," Diagn. Interventional Imaging 94, 487-495 (2013).

125. R. M. S. Sigrist et al., "Ultrasound elastography: review of techniques and clinical applications," Theranostics 7(5), 1303-1329 (2017).
126. R. K. Wang, S. J. Kirkpatrick, and M Hinds, "Phase-sensitive optical coherence elastography for mapping tissue microstrains in real time," Appl. Phys. Lett. 90, 164105 (2007).

127. S. J. Kirkpatrick, R. K. Wang, and D. D. Duncan, "OCT-based elastography for large and small deformations," Opt. Express 14, 11585-11597 (2006)

128. A. S. Khalil et al., "Tissue elasticity estimation with optical coherence elastography: toward mechanical characterization of in vivo soft tissue," Ann. Biomed. Eng. 33, 1631-1639 (2005).

129. G. V. Soest et al., "Robust intravascular optical coherence elastography by line correlations," Phys. Med. Biol. 52, 2445-2458 (2007).

130. J. Rogowska et al., "Optical coherence tomographic elastography technique for measuring deformation and strain of atherosclerotic tissues," Heart 90, 556-562 (2004).

131. H. Ko et al., "Optical coherence elastography of engineered and developing tissue," Tissue Eng. 12, 63-73 (2006).

132. A. G. Bell, "On the production and reproduction of sound by light," Am. J. Sci. s3-20(118), 305-324 (1880).

133. A. G. Bell, "The production of sound by radiant energy," Science os-2(49), 242-253 (1881).

134. C. S. Tainter and A. G. Bell, "Selenium and the photophone," Nature 22, 500-503 (1880).

135. M. Pramanik and L. V. Wang, "Thermoacoustic and photoacoustic sensing of temperature," J. Biomed. Opt. 14(5), 054024 (2009).

136. D. Griffith, Introduction to Electrodynamics, Prentice Hall, New Jersey (1999).

137. S. Blundell and K. M. Blundell, Concepts in Thermal Physics, 2nd ed., OUP, Oxford (2009).

138. M. W. Zemansky and R. H. Dittman, Heat and Thermodynamics, 7th ed., McGraw-Hill Company, Inc., New York (1997).

139. B. T. Cox, J. G. Laufer, and P Beard, "The challenges for quantitative photoacoustic imaging," Proc. SPIE 7177, 717713 (2009).

140. W. R. Hendee and E. R. Ritenour, Medical Imaging Physics, 4th ed., Wiley-Liss, New York (2002).

141. M. S. Singh and P. K. Yalavarthy, "Born-ratio type data normalization improves quantitation in photoacoustic tomography," Proc. SPIE 9040, $90401 \mathrm{~T}$ (2014).

142. B. T. Cox and P. C. Beard, "Fast calculation of pulsed photoacoustic fields in fluids using k-space methods," J. Acoust. Soc. Am. 177(6), 3616-3627 (2005).

143. X. L. Dean-Ben, E. Bay, and D Razansky, "Functional optoacoustic imaging of moving objects using microsecond-delay acquisition of multispectral three-dimensional tomographic data," Sci. Rep. 4, 5878 (2014).

144. H. Goldstein, C. Poole, and J. Safko, Classical Mechanics, 3rd ed., Addison Wesley, New York (2001).

145. T. J. Hall et al., "Phantom materials for elastography," IEEE Trans. Ultrason. Ferroelectr. Freq. Control 44(6), 1355-1365 (1997).

146. R. Cubeddu et al., "A solid tissue phantom for photon migration studies," Phys. Med. Biol. 42, 1971-1979 (1997).

147. J. C. Hebden et al., "A soft deformable tissue-equivalent phantom for diffuse optical tomography," Phys. Med. Biol. 51, 5581-5590 (2006).

Mayanglambam Suheshkumar Singh is as an assistant professor at the School of Physics (SoP), Indian Institute of Science Education and Research Thiruvananthapuram (IISER-TVM), Thiruvananthapuram, Kerala, India. He received his master of science (MSc) degree in physics from Jawaharlal Nehru University (JNU), New Delhi, India, and his PhD from Department of Physics, Indian Institute of Science (IISc), Bangalore, India. The current research interests of his group at IISER-TVM include speckle contrast imaging, photoacoustic imaging (microscopy as well as tomography), and light sheet microscopy.

Anjali Thomas is pursuing her $\mathrm{PhD}$ - under the supervision of MSS-at the School of Physics (SoP), Indian Institute of Science Education and Research Thiruvananthapuram (IISER-TVM), Thiruvananthapuram, Kerala, India. She obtained her master of science (MSc) degree in physics from Kannur University, Kerala, India. She is currently working in design and development of photoacoustic imaging systems (microscopy as well as tomography). 